\title{
Time-resolved characterization and energy balance analysis of implosion core in shock- ignition experiments at OMEGA
}

Cite as: Phys. Plasmas 21, 102709 (2014); https://doi.org/10.1063/1.4898329

Submitted: 02 August 2014 . Accepted: 30 September 2014 . Published Online: 24 October 2014

R. Florido, R. C. Mancini, T. Nagayama, R. Tommasini, J. A. Delettrez, and S. P. Regan
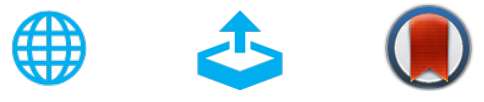

View Online

\section{ARTICLES YOU MAY BE INTERESTED IN}

Direct-drive inertial confinement fusion: A review

Physics of Plasmas 22, 110501 (2015); https://doi.org/10.1063/1.4934714

Understanding reliability and some limitations of the images and spectra reconstructed from a multi-monochromatic $x$-ray imager

Review of Scientific Instruments 86, 113505 (2015); https://doi.org/10.1063/1.4935828

The physics basis for ignition using indirect-drive targets on the National Ignition Facility

Physics of Plasmas 11, 339 (2004); https://doi.org/10.1063/1.1578638

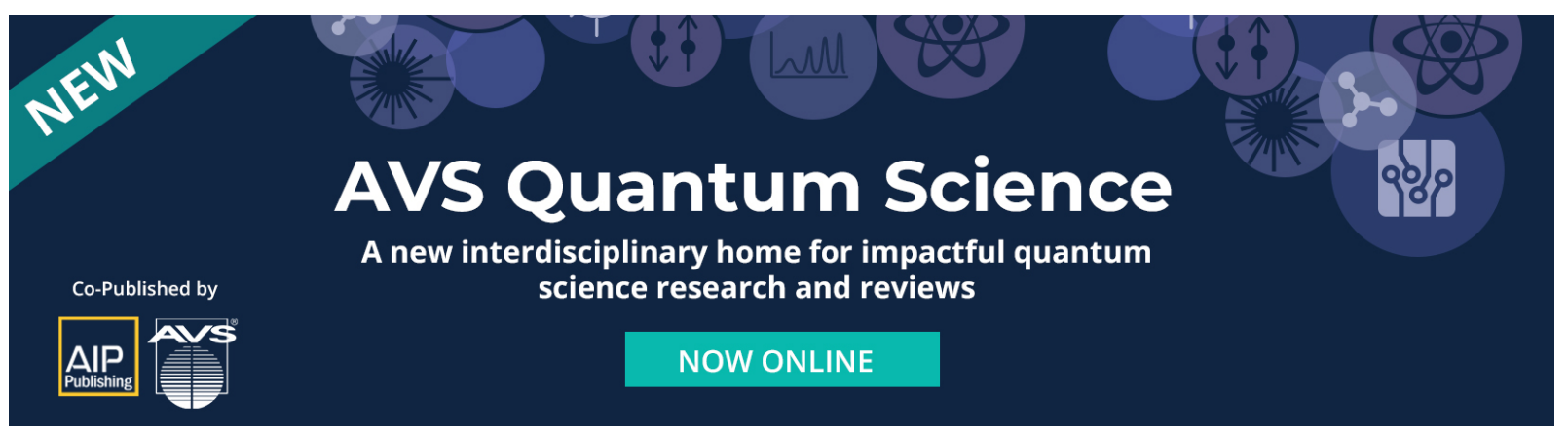




\title{
Time-resolved characterization and energy balance analysis of implosion core in shock-ignition experiments at OMEGA
}

\author{
R. Florido, ${ }^{1, a)}$ R. C. Mancini, ${ }^{1}$ T. Nagayama, ${ }^{1, b)}$ R. Tommasini, ${ }^{2}$ J. A. Delettrez, ${ }^{3}$ \\ and S. P. Regan ${ }^{3}$ \\ ${ }^{1}$ Department of Physics, University of Nevada, Reno, Nevada 89557, USA \\ ${ }^{2}$ Lawrence Livermore National Laboratory, Livermore, California 94550, USA \\ ${ }^{3}$ Laboratory for Laser Energetics, University of Rochester, Rochester, New York 14623, USA
}

(Received 2 August 2014; accepted 30 September 2014; published online 24 October 2014)

Time-resolved temperature and density conditions in the core of shock-ignition implosions have been determined for the first time. The diagnostic method relies on the observation, with a streaked crystal spectrometer, of the signature of an Ar tracer added to the deuterium gas fill. The data analysis confirms the importance of the shell attenuation effect previously noted on time-integrated spectroscopic measurements of thick-wall targets [R. Florido et al., Phys. Rev. E 83, 066408 (2011)]. This effect must be taken into account in order to obtain reliable results. The extracted temperature and density time-histories are representative of the state of the core during the implosion deceleration and burning phases. As a consequence of the ignitor shock launched by the sharp intensity spike at the end of the laser pulse, observed average core electron temperature and mass density reach $T \sim 1100 \mathrm{eV}$ and $\rho \sim 2 \mathrm{~g} / \mathrm{cm}^{3}$; then temperature drops to $T \sim 920 \mathrm{eV}$ while density rises to $\rho \sim 3.4 \mathrm{~g} / \mathrm{cm}^{3}$ about the time of peak compression. Compared to 1D hydrodynamic simulations, the experiment shows similar maximum temperatures and smaller densities. Simulations do not reproduce all observations. Differences are noted in the heating dynamics driven by the ignitor shock and the optical depth time-history of the compressed shell. Time-histories of core conditions extracted from spectroscopy show that the implosion can be interpreted as a two-stage polytropic process. Furthermore, an energy balance analysis of implosion core suggests an increase in total energy greater than what $1 \mathrm{D}$ hydrodynamic simulations predict. This new methodology can be implemented in other ICF experiments to look into implosion dynamics and help to understand the underlying physics. (C) 2014 AIP Publishing LLC. [http://dx.doi.org/10.1063/1.4898329]

\section{INTRODUCTION}

Present work reports on the time-resolved measurements and energy balance inferred from spectroscopic diagnosis of implosion core in shock-ignition (SI) experiments at OMEGA. $\mathrm{SI}^{1}$ is an alternative direct-drive approach expected to achieve ignition using only a third of the driver energy needed in the conventional hot spot scheme. It has aroused considerable interest in the ICF community and motivated studies for implementation at existing and planned ICF facilities including OMEGA, ${ }^{2} \mathrm{NIF}^{3}{ }^{3} \mathrm{LMJ},{ }^{4}$ and HiPER. ${ }^{5}$ Theoretical models and numerical studies on different aspects including target robustness, design and scaling, ${ }^{6-13}$ spectroscopic modeling, ${ }^{14}$ electron transport, ${ }^{15}$ laser-plasma interaction, ${ }^{16}$ novel driving strategies, ${ }^{17,18}$ etc. have been published over the last few years, thus establishing SI as a very active research area. Nevertheless, only few experimental studies have been reported. Initial SI experiments performed at the OMEGA laser facility successfully tested the concept, ${ }^{2}$ and performance improvements were reported using a new configuration of the laser

\footnotetext{
${ }^{a}$ Permanent address: Departamento de Física, Universidad de Las Palmas de Gran Canaria, 35017 Las Palmas de Gran Canaria, Spain; Electronic mail: ricardo.florido@ulpgc.es

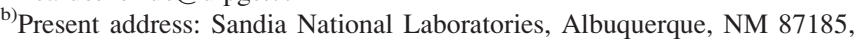
USA.
}

driver. ${ }^{19}$ In parallel, the feasibility of space- and timeintegrated spectroscopy for a simultaneous diagnosis of both core and shell conditions in Ar-doped SI implosions was demonstrated, emphasizing the importance of the shell attenuation effect for a correct interpretation and analysis of the data. ${ }^{20}$ Also, experiments using planar target for studying laser-plasma interaction and shock propagation relevant to SI were conducted at LULI-LIL, ${ }^{16}$ LULI, $^{21,22}$ Shenguang III prototype, ${ }^{23}$ and OMEGA. ${ }^{24}$ Recent reviews of the concept principles and issues can be found in Refs. 25 and 26.

In ICF experiments, diagnosis of core plasma temperature and density conditions is critical to assess the performance of implosion hydrodynamics. X-ray spectroscopy has proved to be a powerful technique to investigate different aspects of laser-fusion experiments ${ }^{27}$ including target preheat due to fast electrons by means of $\mathrm{K} \alpha$ emission spectroscopy, ${ }^{28,29}$ average electron temperature and density in implosion cores of directand indirect-drive implosions including Stark-broadened Kand L-shell line emissions, ${ }^{30-38}$ spatial profiles of temperature and density from the analysis of x-ray spectra and narrowband images, ${ }^{39-41}$ asymmetry measurements of plasma conditions spatial profiles from pinhole space-resolved spectra, ${ }^{42}$ and the development of a polychromatic tomography method for the extraction of the 3D spatial structure of implosion core plasmas. $^{43}$ 
Here, we extend the application of x-ray spectroscopy to ICF experiments by presenting the first time-resolved measurements of core density and temperature in SI implosions. The potential application of x-ray spectroscopy to SI was motivated by modeling results, ${ }^{14}$ and subsequently applied to the diagnosis of both core and compressed shell conditions from the analysis of the space- and time-integrated Ar tracer spectrum. ${ }^{20}$ This article builds on this previous work and presents the analysis of time-resolved measurements. The study permits to track the onset of an attenuation effect in the compressed shell and its impact on the emergent intensity distribution of the Ar K-shell lines. It will be shown that, provided the attenuation effect is taken into account, meaningful information can be unfolded from the data, making possible the determination of temperature and density timehistories representative of the state of the core during the deceleration and burning phases.

This work is also in line with the report published by the U. S. National Nuclear Security Administration ${ }^{44}$ at the end of the National Ignition Campaign. ${ }^{45}$ The report emphasizes the importance of research on alternative concepts to hotspot approach and the improvement of diagnostic techniques for testing large-scale simulation's prediction and a better understanding of ICF physics. It is noted that the standard set of diagnostics of integrated implosion experiments mainly consists of time-integrated particle measurements that are then compared with the corresponding global quantities from simulations, which in turn depend on multiple physics models and phenomena. Comparisons between data and simulations can show global discrepancies, but reasons of discrepancies or the way to improve the models are difficult to isolate. In this regard, the time-resolved analysis discussed in this work provides new diagnostic information that allows to track the core conditions during a critical stage of the implosion.

From the quantities directly extracted from the analysis, i.e., core temperature and density, and the assumption of an equation-of-state, the pressure time-history can be determined. This opens up a window in the dynamics of SI implosion cores. The thermodynamic characteristics of the core compression were explored and it was found to be representative of a two-stage polytropic process. Furthermore, a study of the core energy balance during the deceleration phase was performed and we estimated the energy losses due to thermal conduction and bremsstrahlung, and $P d V$-work done by the ablator on the fuel, to then determine change in total energy. These results are valuable for studying the performance of ICF implosions. Although the analysis was applied to SI implosions, we emphasize that the method is approach independent and can be employed to study direct/ indirect-drive or polar-drive experiments.

This paper is organized as follows. Section II describes the experimental setup and the recorded data. Section III considers the 1D hydrodynamic simulation of the experiment, and the time-correlation between time-resolved measurements and simulation. Sec. IV introduces the physics model employed in the analysis, and Sec. V discusses the results of the time-resolved spectroscopic analysis and the comparison with the simulation. Section VI presents a thermodynamic interpretation of the deceleration and burning phases in terms of a polytropic process and an analysis of the energy balance of the implosion core. Finally, conclusions are presented in Sec. VII.

\section{EXPERIMENT DETAILS}

The experiments were performed at the OMEGA Laser Facility ${ }^{46}$ using the 60 -beam configuration for driving symmetric implosions. Targets consisted of spherical plastic shells that had an internal radius of $394 \mu \mathrm{m}$, a wall thickness of $40 \mu \mathrm{m}$, and an outer aluminum coating of $0.1 \mu \mathrm{m}$ for sealing purposes. They were filled with $20 \mathrm{~atm}$ of $\mathrm{D}_{2}$ and $0.072 \mathrm{~atm}$ of Ar, which was used as a tracer for spectroscopic diagnosis of the core. Relaxation adiabat shaping ${ }^{47}$ laser pulses of $\sim 18.6 \mathrm{~kJ}$ UV laser energy were used to drive the implosions. The adiabat is an indicator of the entropy value associated to a target trajectory in the pressure-density space. Laser beams were smoothed with distributed phase plates $^{48,49}$ and polarization smoothing using birefringent wedges. ${ }^{5-52}$ The pulse shape (Fig. 1) comprised of a $80 \mathrm{ps}$ FWHM Gaussian picket, which sets the adiabat of the implosion, followed by a $1.5 \mathrm{~ns}$ long main drive with a sharp intensity spike at the end to launch the ignitor shock wave. To optimize the implosion performance, both the picket and the spike must be properly timed, so that the ignitor shock meets the outward moving shock caused by the reflection of the main shock in the center, at the inner region of the dense shell. This collision originates a new shock wave moving inward that leads to further compression of the fuel and, ideally, boosts it up to ignition. In these experiments, picket and spike delays were set to $\sim 0 \mathrm{~ns}$ and $\sim 2.8 \mathrm{~ns}$, respectively, which were the optimal values found in SI spherical implosions using plastic-shell targets filled with $25 \mathrm{~atm}$ of $\mathrm{D}_{2}{ }^{2}{ }^{2}$

The diagnostics fielded in these experiments included a neutron time-of-flight detector for total neutron yield measurements $^{53}$ and a nuclear temporal diagnostic (NTD) ${ }^{54}$ that provided the fusion reaction-rate time-history of the implosions. An x-ray spectrometer (XRS1) equipped with an ADP (ammonium dihydrogen phosphate) crystal and spectral

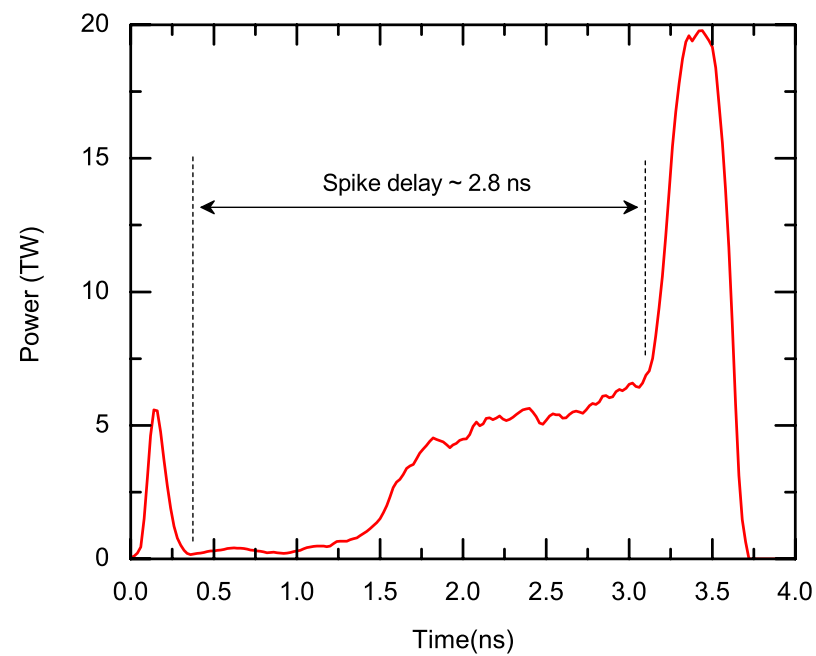

FIG. 1. Laser pulse shape used in the spherical shock-ignition implosion experiments performed at OMEGA. 
resolution power of $\lambda / \Delta \lambda \approx 1200$ recorded the time- and space-integrated $\mathrm{x}$-ray line spectrum, and a streaked spectrometer (SSCA), which utilized a RbAP (rubidium acid phthalate) crystal for dispersing the $\mathrm{x}$-rays onto a Au photocathode with sweep speed of $\sim 115 \mathrm{ps} / \mathrm{mm}$ and $\lambda / \Delta \lambda \approx 500$, was used to measure the time-resolved $\mathrm{Ar}$ emission from the core through the collapse of the implosion.

As an illustration, Fig. 2 displays the image data recorded by the streak camera for OMEGA shot 53258. A portion of the streaked camera's photocatode was blocked, providing a wavelength fiducial to establish the orientation of the streak axis and a reference for undoped experiments. Details about data processing can be found in Refs. 37 and 55. The time axis was set by performing a simultaneous time-correlation between broad band x-ray emission signals extracted from the data and that predicted by a $1 \mathrm{D}$ hydrodynamic simulationsee discussion below. The streaked data shows, early in time, the continuum radiation emission from the plasma blow-off. Late in time-i.e., at the collapse of the implosion-the characteristic Ar line emission and compression continuum, with durations of $\sim 250 \mathrm{ps}$ and $\sim 200 \mathrm{ps}$, respectively, are observed. The Ar K-shell line emission observed with SSCA begins at $\sim 4.3 \mathrm{~ns}, \sim 0.6 \mathrm{~ns}$ after the laser is shut off, spanning the photon energy range from $3400 \mathrm{eV}$ to $4400 \mathrm{eV}$. Several transitions of He- and H-like Ar ions have been identified and labeled, namely, $\mathrm{He}-\beta \quad\left(1 s 3 p \rightarrow 1 s^{2}\right), \quad \mathrm{He}-\gamma \quad\left(1 s 4 p \rightarrow 1 s^{2}\right), \quad \mathrm{Ly}-\beta$ $(3 p \rightarrow 1 s)$, and Ly- $\gamma(4 p \rightarrow 1 s)$. Weaker, and sometimes heavily blended with parent lines, satellite line transitions arising from autoionizing states in He- and Li-like $\mathrm{Ar}$ ions are also present in the data. As the implosion proceeds and the density increases, line features broaden as a consequence of the Starkbroadening effect.

Sampling the image in Fig. 2 with a rectangular horizontal or vertical box will produce a time history of radiation emitted within a given photon energy range or a time-

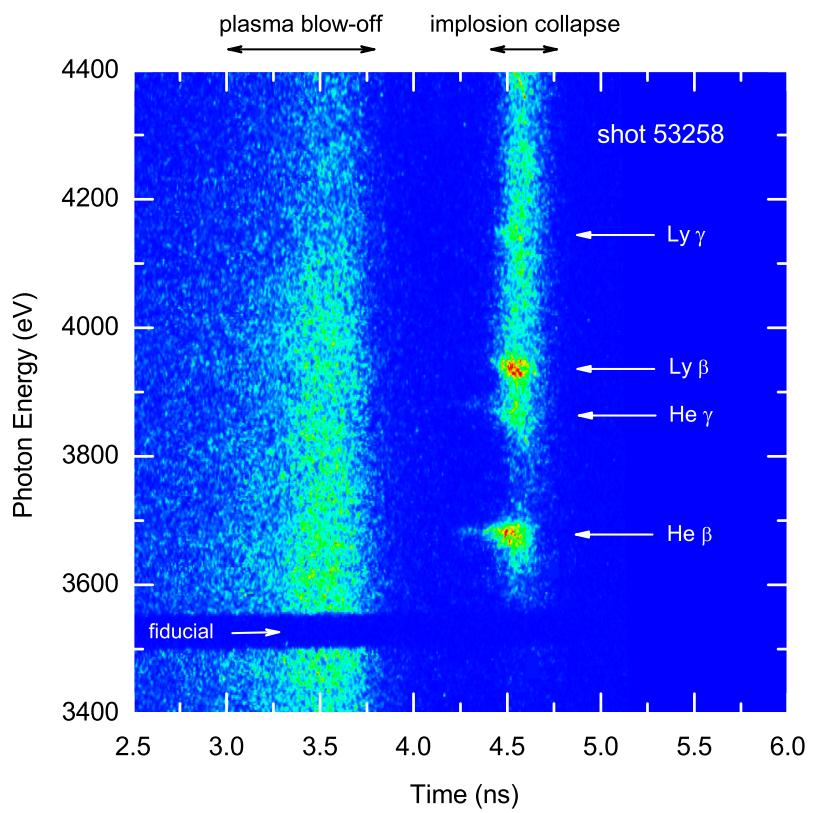

FIG. 2. Streaked data from SSCA for OMEGA shot 53258. Emissions from the coronal plasma blow-off, and compression continuum and Ar K-shell lines are observed. resolved spectrum, respectively. As an example, two time-resolved spectra characteristic of the same short timeintervals are displayed in Fig. 3. The good comparison of spectra from nominally identical shots (53258 and 53259) for equivalent times gives evidence of the reproducibility of the Ar emission observation.

Furthermore, the time-integrated spectrum obtained from the SSCA streaked data was compared to the spectrum measured by the XRS1 crystal spectrometer-see Fig. 4. The comparison between these two independent measurements shows consistency and provides additional confidence on the SSCA data processing.

\section{HYDRODYNAMIC SIMULATIONS, TIME- CORRELATION, AND NEUTRON MEASUREMENTS}

The 1D Lagrangian hydrodynamics code LILAC $^{56}$ was used to simulate the experiments. The purpose of simulations was two-fold: First, the analysis of synthetic spectra obtained from post-processing the hydrodynamic results was used to validate the spectroscopic method; and, second, simulations were tested by comparing with the results of the data analysis. Two types of calculations were performed: (1) a LILAC-
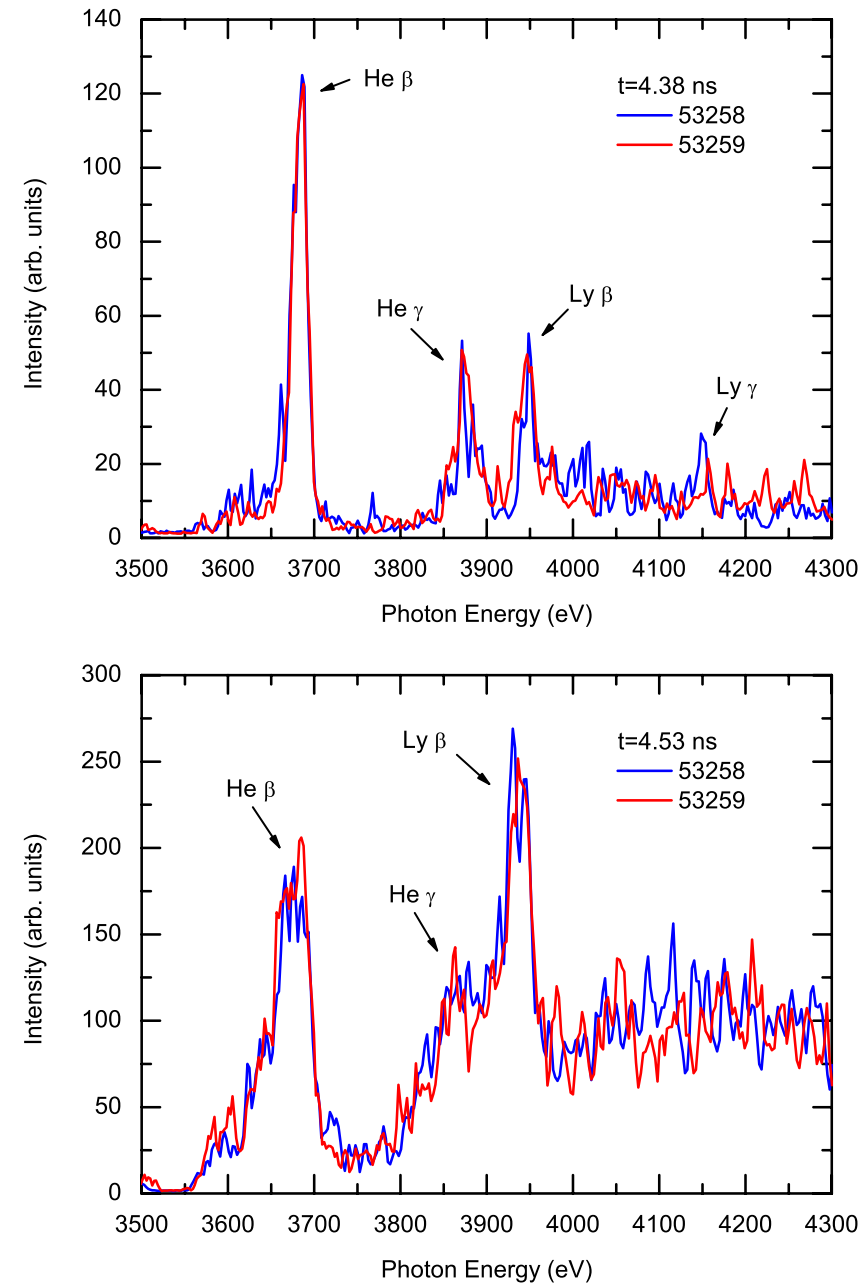

FIG. 3. Comparison of time-resolved spectra from two nominally identical experiments for equivalent times: (top) $t=4.38 \mathrm{~ns}$, and (bottom) $t=4.53 \mathrm{~ns}$. Each spectrum is characteristic of a $75 \mathrm{ps}$ time interval centered at the indicated time. 


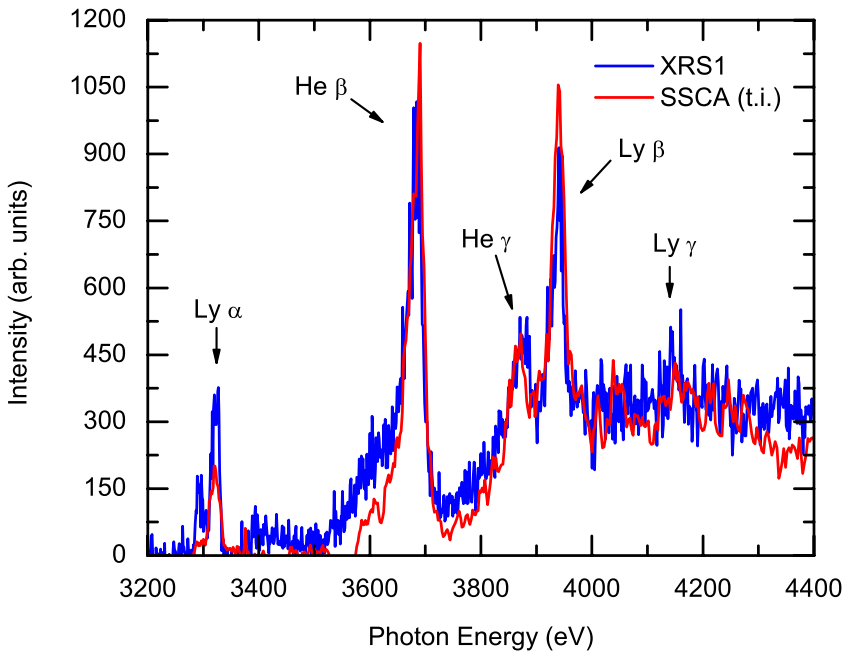

FIG. 4. XRS1 spectrum compared with time-integrated SSCA data for OMEGA shot 53259.

standard simulation in which the heat flux model included a flux-limiting factor $f=0.06$ and no laser cross beam energy transfer effect, and (2) a LILAC-enhanced simulation that considered non-local thermal transport ${ }^{57}$ as well as the effect of cross beam energy transfer (CBET). ${ }^{58}$ Other than these differences, the physics model was the same in both simulations. The comparison of results including and not including Ar in the core shows that the temperature decreases due to the presence of the dopant. This is because of the radiative cooling effect of the Ar emission, which also causes a reduction of the total neutron yield. ${ }^{59}$ Despite this, simulations show that the presence of the Ar dopant does not significantly alter the hydrodynamic evolution, whose qualitative behavior remains largely unchanged. This point is illustrated in Fig. 5 where the position of Lagrangian coordinates versus time from LILAC-standard simulation with and without $\mathrm{Ar}$ tracer are shown during the final stage of the implosion. The Ar-doped target is compressed to a minimum core radius of

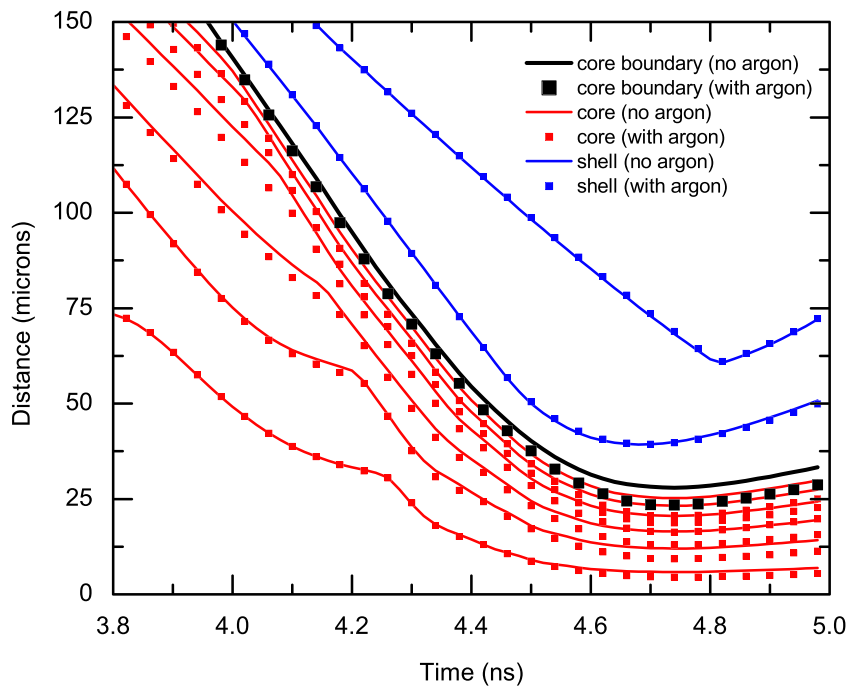

FIG. 5. Position of selected core and shell Lagrangian coordinates versus time for LILAC-standard simulations considering both Ar-doped and undoped targets.
$23 \mu \mathrm{m}$ compared to $28 \mu \mathrm{m}$ of the undoped case. In both cases, the effect of the ignitor shock launched by the laser's intensity spike reaches the core at about $t=4 \mathrm{~ns}$.

The hydrodynamic behavior predicted by LILACstandard and LILAC-enhanced simulations-both including the Ar dopant in the core-is discussed next. The influence of the different compression waves is seen in Fig. 6, where the velocity of the core boundary Lagrangian coordinate from both simulations is plotted versus time; the laser pulse shape is also displayed. Differences are noted between simulations, but, in both cases, the implosion core boundary continues being accelerated inwards until $\sim 4.2 \mathrm{~ns}$. After that, the core boundary starts to decelerate. This point, therefore, can be taken as the beginning of the deleceration phase, which lasts until the time of maximum compression where the core boundary velocity is zero (i.e., minimum core radius): $t=4.72 \mathrm{~ns}$ for LILAC-standard and $t=4.86 \mathrm{~ns}$ for LILAC-enhanced. Both simulations have similar characteristics, with LILAC-enhanced achieving smaller maximum inward velocity and having a longer deceleration phase. Also, the arrival of the ignitor shock at the core boundary before the beginning of the deceleration phase suggests that, according to simulations, the rebound and the ignitor shocks do not meet in the inner region of the shell, but rather in the outer portion of the core.

The time-history of temperature and density spatial profiles computed by LILAC-standard and LILAC-enhanced were postprocessed to obtain space integrated and either timeresolved or time-integrated Ar spectra including the boundbound, bound-free, and free-free contributions. The emergent intensity distribution was computed by integrating the radiation transport equation along chords in the target, one photon energy at a time, in the photon energy range relevant for the Ar K-shell lines. Then, individual chord contributions were integrated in volume to obtain the space-integrated spectrum observed along a given line-of-sight. ${ }^{14,20}$ To this end, the required temperature-, density-, and frequency-dependent emissivity and opacity of the core and shell in the

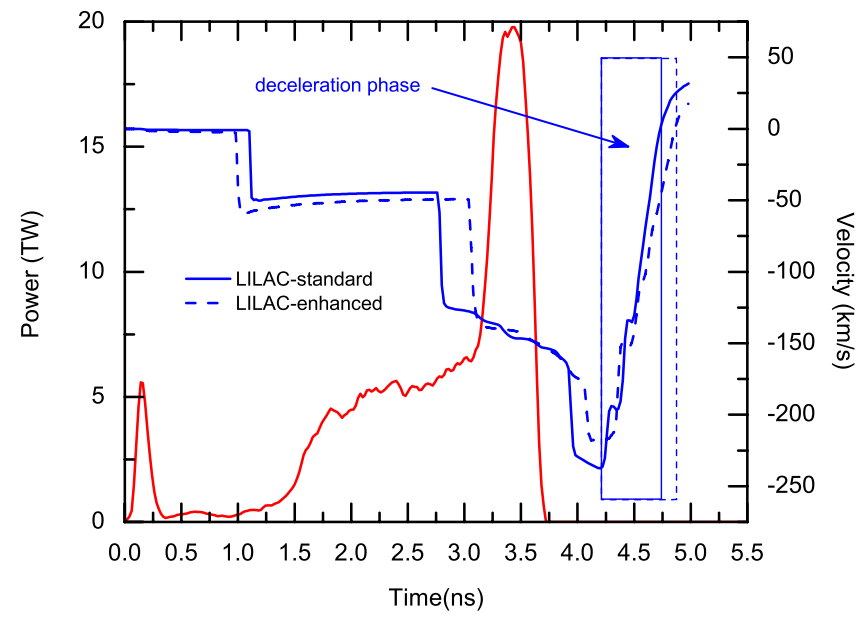

FIG. 6. Laser pulse shape and velocity of Lagrangian coordinate representing the core boundary from LILAC-standard and LILAC-enhanced simulations. A negative velocity indicates that the boundary is moving inwards. The rectangular boxes indicate the time-interval corresponding to the deceleration phase predicted by each simulation. 
$3000-4500 \mathrm{eV}$ photon energy range were computed with the collisional-radiative atomic kinetics model ABAKO. ${ }^{60}$

From the last results, the space and photon energy integrated radiation power can be calculated - the integration was performed over the photon energy interval from 3400 to $4400 \mathrm{eV}$ to be consistent with the SSCA observation. The computed radiation power is now used to set the timing of the streaked data. The laser pulse signal and the computed radiation power are time-correlated since the first one is an input of the simulation-we consider $t=0 \mathrm{~ns}$ when the laser is turned on. Then, from the SSCA data, we extract the experimental radiation power, which so far has an arbitrary initial time. A reference time, preferably associated to a physics event, is needed in order to set the time-axis of the experiment. Early in time, the radiation power corresponds to the emission of the plasma blow-off, and this is expected to follow closely the laser pulse shape. Thus, we chose the time of blow-off emission peak as the reference event and match the computed and experimental radiation power peak times at this point. In this way, all signals are on the same time-axis and comparison between simulations and data is now possible. It is noted that no other condition (e.g., on signal shapes or relative timing of other events) is imposed.

Time-correlated computed and experimental radiation power, and the laser pulse shape are plotted in Fig. 7. It is seen how, early in time, the computed and experimental radiation power follow the laser pulse shape with a small delay. In particular, the intensity spike in the laser occurs at $t=3.42 \mathrm{~ns}$ while the experimental blow-off emission peaks at $t=3.52 \mathrm{~ns}$. Computed blow-off radiation powers based on LILAC-standard and LILAC-enhanced simulations match the experimental measurement behavior well, but LILACstandard approximates the earlier blow-off x-ray emission better than LILAC-enhanced. Once the laser pulse is turned off, the second peak in the experimental radiation power, centered at $t=4.60 \mathrm{~ns}$, is due to the $\mathrm{Ar}$ and compression continuum emissions characteristic of the collapse of the implosion. The calculation based on LILAC-standard matches the

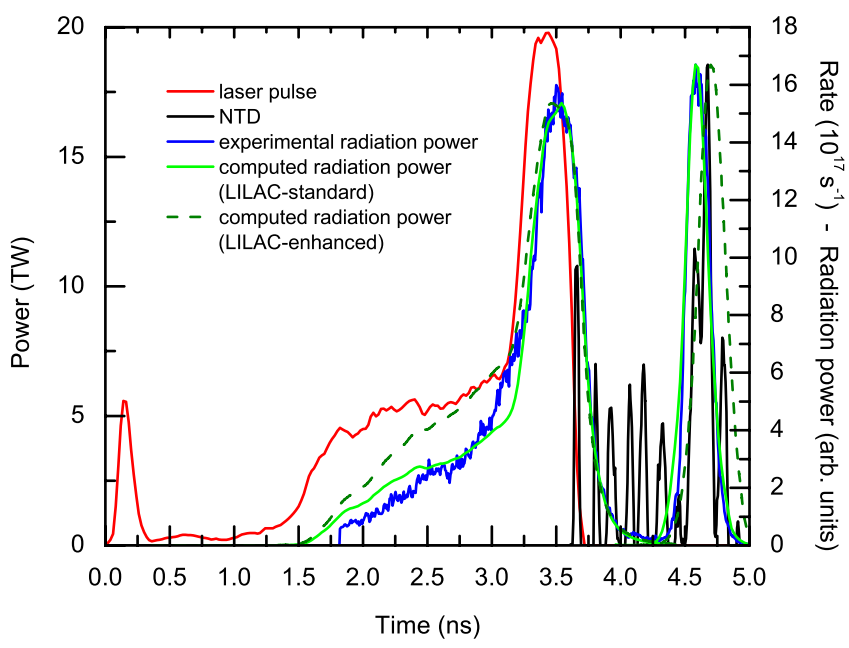

FIG. 7. Laser pulse shape, experimental and computed radiation power (based on LILAC-standard and LILAC-enhanced simulations) timehistories, and neutron rate history from the NTD diagnostic. The uncertainty in the absolute timing is $\pm 50 \mathrm{ps}$. timing of this second peak well $(t=4.58 \mathrm{~ns})$, while LILACenhanced's second peak occurs a bit later, at $t=4.70 \mathrm{~ns}$. Also shown in Fig. 7 is the experimental burn rate (i.e., neutron production rate) time-history recorded with the NTD diagnostic, which has a peak at $t=4.67 \mathrm{~ns}$; this is the bang time of the implosion. The bang times predicted by the LILAC-standard and LILAC-enhanced simulations were $t=4.58 \mathrm{~ns}$ and $t=4.70 \mathrm{~ns}$, respectively. Thus, while LILACstandard approximates better the timing of the $\mathrm{Ar}$ and continuum compression $\mathrm{x}$-ray emission, LILAC-enhanced does it for the bang time. We emphasize that the overlapping between the experimental Ar and continuum compression Xray emission and burn rate peaks indicates that the core conditions extracted from the spectroscopy include the state of the implosion core during the burn at the end of the deceleration phase.

For both shots, 53258 and 53259, the measured neutron yield was $4.4( \pm 0.4) \times 10^{8}$. The ratio of the measured primary neutron yield to that predicted by $1 \mathrm{D}$ simulations, i.e., yield-over-clean (YOC), was $\sim 6 \%$ for LILAC-standard and $\sim 22 \%$ for LILAC-enhanced. In this regard, when non-local heat transport and CBET effects are considered, the 1D simulation agrees better with experiment.

\section{SPECTROSCOPIC MODEL}

The potential application of x-ray spectroscopy to SI implosions was initially suggested based on modeling results, ${ }^{14}$ and subsequently applied to the diagnosis of both core and compressed shell conditions from the analysis of the space- and time-integrated Ar tracer spectrum. ${ }^{20}$ The method relied on the simultaneous observation over a broad photon energy range of the Ar line emission and the attenuation and self-emission effects of the compressed shell confining the core. A detailed discussion of the emergent intensity distribution in the photon energy range from $3000 \mathrm{eV}$ to $4400 \mathrm{eV}$ was given in Ref. 20. Briefly, the observed timeintegrated spectrum showed an attenuation effect not seen in other Ar-doped ICF experiments. The attenuation was particularly severe in the range from $3000 \mathrm{eV}$ to $3400 \mathrm{eV}$. The usually strong $\mathrm{He}-\alpha\left(1 s 2 p \rightarrow 1 s^{2}\right)$ and $\mathrm{Ly}-\alpha(2 p \rightarrow 1 s)$ line emissions appeared among the weakest lines in the spectrum. To a lesser extent, the attenuation also changed the relative intensity of the He- $\beta$ and Ly- $\beta$ lines. The effect was interpreted in terms of the radiation absorption in the compressed shell confining the core, due to inverse bremsstrahlung (freefree) and photoionization (bound-free) processes. It was shown that the simultaneous observation over the broad photon energy range from $3000 \mathrm{eV}$ to $4400 \mathrm{eV}$ of the core $\mathrm{Ar}$ line emission and the attenuation and self-emission effects of the compressed shell confining the core had enough information to extract spatially averaged temperature and density conditions in both core and compressed shell. The spectroscopic analysis also provided an estimate of the target's areal density.

In the experiments reported here, the data recorded by the streak camera were in the photon energy range from $3400 \mathrm{eV}$ to $4400 \mathrm{eV}$. This narrower range prevents the application of the method previously employed with time- 
integrated data ${ }^{14,20}$ to the time-resolved spectra considered here. Nevertheless, an alternative technique can still be used to diagnose core conditions.

The spectroscopic model of the emergent intensity distribution is calculated as

$$
I_{\nu}=I_{\nu}^{c} e^{-\tau_{\nu}^{s}}
$$

In this formula, $\nu$ is the photon frequency, $I_{\nu}^{c}$ represents the intensity distribution of the radiation emitted by a spherical plasma source of uniform electron temperature $T_{e}$ and density $N_{e}$ that is used to model the implosion core. This intensity distribution is obtained by integrating the radiation transport equation along chords in the source one photon energy at a time and then adding up the contributions from all the chords. For the case of a uniform sphere, the integral is analytical and it is given by ${ }^{61}$

$$
I_{\nu}^{c}=\pi R^{2} \frac{\varepsilon_{\nu}^{c}}{\kappa_{\nu}^{c}}\left[1+\frac{e^{-2 \kappa_{\nu}^{c} R}}{\kappa_{\nu}^{c} R}-\frac{\left(1-e^{-2 \kappa_{\nu}^{c} R}\right)}{2\left(\kappa_{\nu}^{c} R\right)^{2}}\right],
$$

where $\varepsilon_{\nu}^{c}$ and $\kappa_{\nu}^{c}$ stand for the temperature, density, and photon energy dependent emissivity and opacity of the core, respectively. The exponential factor, $e^{-\tau_{\nu}^{s}}$, in Eq. (1) accounts for the transmission of the core emission through an effective slab representing the attenuation in the compressed shell. Since the absorption effect in the shell is due to photoionization and inverse bremsstrahlung, the leading photon energy dependence can be factored out and the optical depth written as $\tau_{\nu}^{s}=\frac{a}{(h \nu)^{3}}$. The attenuation parameter " $a$ " is a function of the shell conditions, but, since there was not enough information in the observed spectral range to unfold this dependence, it was considered a free parameter of the model. The spectroscopic analysis was performed by means of a weighted least-square minimization procedure where the experimental spectrum was fitted according to Eq. (1). Before comparing with data, the theoretical spectrum is convolved with the instrumental function to account for the finite spectral resolution of the instrument. An exhaustive search on the three model parameters $a, T_{e}$, and $N_{e}$ yielded a single, absolute minimum from which the values of the parameters were extracted. A systematic application of this procedure to a sequence of time-resolved spectra resulted in the extraction of the time history of electron temperature $T_{e}$ and density $N_{e}$ in the core. The mass density $\rho$ is computed from $N_{e}$ and the corresponding ionization balance. We emphasize that the spectroscopic model is independent of the hydrodynamic simulation. Hence, the data analysis results are also useful to compare with the simulation.

The photon energy range for analysis spans from $3500 \mathrm{eV}$ to $4300 \mathrm{eV}$, and it relied on the information encoded in $\mathrm{Ar} \mathrm{He}-\beta, \mathrm{He}-\gamma, \mathrm{Ly}-\beta$ and $\mathrm{Ly}-\gamma$ lines, and their associated Li- and He-like satellite transitions as well as bound-free contributions involving fully-stripped, H-like and He-like ions. Most previous applications of Ar K-shell spectroscopy for diagnosing implosion cores mainly focused on $\mathrm{He}-\beta$, He$\gamma, \mathrm{Ly}-\beta$, and their satellites. Here, the photon energy range was extended up to $4300 \mathrm{eV}$, thus further including $\mathrm{Ly}-\gamma$ and bound-free contributions. Thus, by considering a wider photon energy range, the spectroscopic analysis relied on more information and hence was more constrained. ${ }^{55}$

Photon-energy dependent emissivities and opacities needed for the analysis were calculated with population number densities from the collisional-radiative atomic kinetics model ABAKO. ${ }^{60}$ For this application, an ABAKO model for Ar was constructed that included up to 4592 energy levels (1 fully stripped, $100 \mathrm{H}$-like, $352 \mathrm{He}$-like, 519 Li-like, 644 Be-like, 1299 B-like, and 1677 C-like Ar). Energy levels and radiative line transition rates were computed using the atomic structure code $\mathrm{FAC}^{62}$ including unresolved transition arrays $^{63}$ and configuration interaction corrections. The calculations took into account all nonautoionizing and autoionizing states characterized by principal quantum numbers consistent with the continuum lowering, so that the actual final number of levels included in the calculation depended on the plasma conditions of each case. Moreover, the lowering of the ionization potential of an Ar ion compared to the value for the unperturbed, isolated case was calculated including the effect of the deuterium-Ar mixture environment in the core, as discussed in Ref. 55. Collisional-radiative atomic kinetic calculations for the range of plasma conditions achieved in the core confirm that time scales of dominant atomic processes are much smaller than typical hydrodynamic evolution times, so that timedependent effects in the atomic kinetics were not significant.

Radiation transport effects on level population kinetics due to line trapping in the plasma were taken into account via escape factors. ${ }^{64}$ The line profile used in the escape factor calculation was a Voigt function in which the width of the Lorentzian contribution considered natural and a Stark width based on detailed line shape calculations, and the width of the Gaussian was determined by thermal Doppler broadening. On the other hand, to transport the radiation through the plasma, detailed Stark-broadened line profiles were used in the Ar emissivity and opacity-see Eq. (2). A database of line profiles was computed for line transitions arising from non-autoionizing as well as autoionizing states taking into account the broadening effects due to both plasma electrons and ions, as well as natural and Doppler broadening. ${ }^{65,66}$ Ion microfield distribution functions were computed with the APEX model assuming equal electron and ion temperatures. ${ }^{67}$ Also, since deuterium ions comprised the majority of the perturbing ions, ion dynamics effects were considered in the Stark line broadening calculation according to the formalism discussed in Ref. 35. The calculation of radiative recombination rates in the atomic kinetics employed the Kramers' approximation for the photoionization cross section; ${ }^{68}$ however, the bound-free contributions to the emissivity and opacity for the radiation transport through the core were determined from analytical fits to accurate photoionization cross sections computed with an atomic scattering code. ${ }^{69}$ In particular, the following bound-free contributions within the spectral range of analysis were included in the model: from fully-stripped ion into $\mathrm{H}$ like ground state, from $\mathrm{H}$-like ground state into He-like ground state, and from H-like $n l$ excited states into He-like 1s $n l$ excited states, up to $n=4$. Additionally, a plasma broadening effect on the bound-free emissivity and opacity 
edges was included according to the approximation discussed in Ref. 70.

\section{TIME-RESOLVED ANALYSIS}

First, the analysis method was tested with synthetic spectra obtained by post-processing the hydrodynamic simulation spatial profiles. Figure 8 shows the comparisons between synthetic spectra from LILAC-standard and best model fits at two different times representative of the spectroscopic analysis time-interval. Early in the deceleration phase, the lines are well resolved and the bound-free emission overlapping with the Ly- $\gamma$ is weak. Later, larger Stark broadening characteristic of a denser plasma blends the $\mathrm{He}-\gamma$ and Ly- $\beta$ lines, and the bound-free emission increases. This trend in the synthetic spectra is similar to that observed in the data shown in Fig. 3. The model given by Eq. (1) approximates well the synthetic spectra, and the temperature and density values extracted from the analysis for each spectrum fall within the range of the corresponding spatial profiles. The analysis was performed over the photon energy interval from $3500 \mathrm{eV}$ to $4300 \mathrm{eV}$, which is the same as the one used
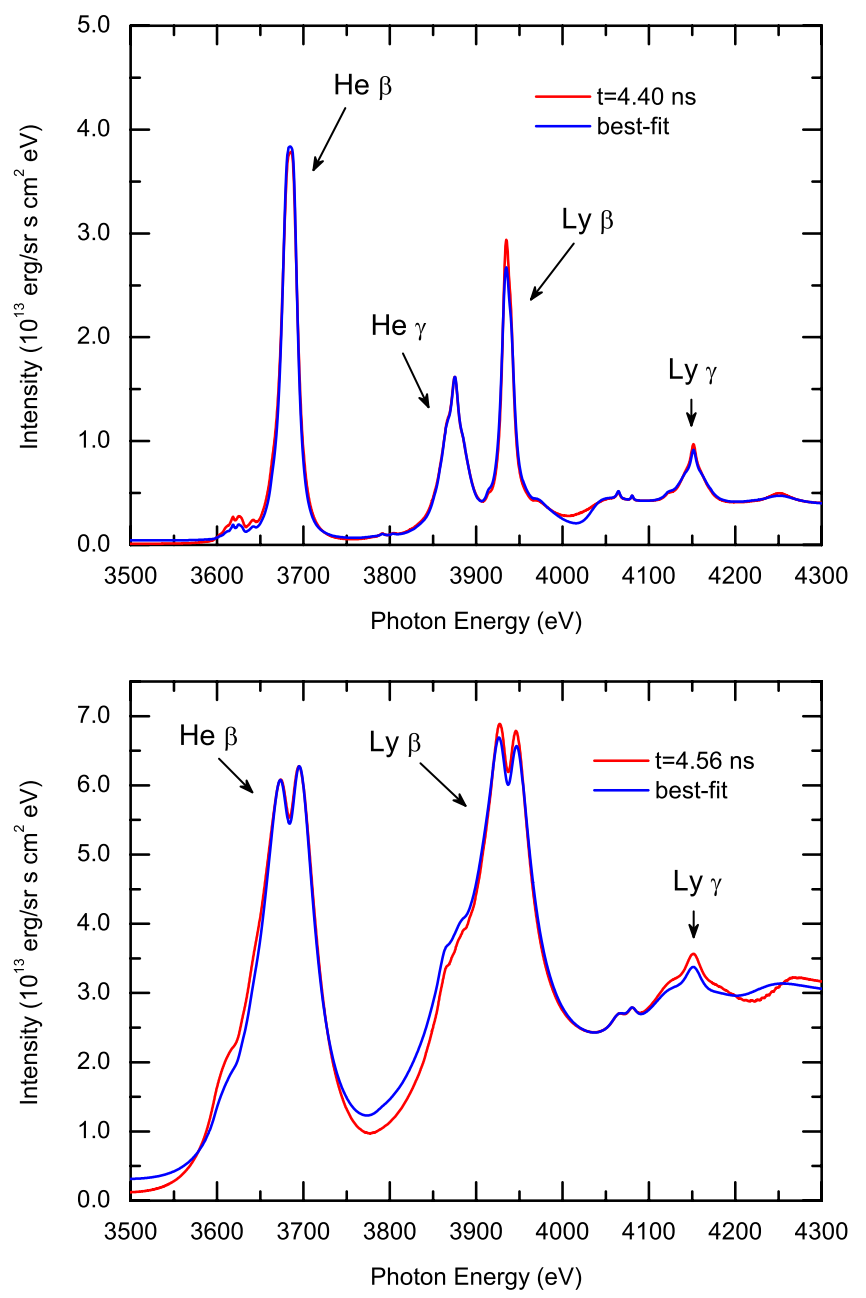

FIG. 8. Spectroscopic analysis of time-resolved synthetic spectra from LILAC-standard over the photon energy range from $3500 \mathrm{eV}$ to $4300 \mathrm{eV}$. Core conditions of model best-fit were: for $t=4.40 \mathrm{~ns}, T_{e}=1008 \mathrm{eV}$, $N_{e}=3.2 \times 10^{23} \mathrm{~cm}^{-3}, \rho=1.1 \mathrm{gcm}^{-3}$; and for $t=4.56 \mathrm{~ns}, T_{e}=1090 \mathrm{eV}$, $N_{e}=2.0 \times 10^{24} \mathrm{~cm}^{-3}, \rho=6.7 \mathrm{gcm}^{-3}$. in the analysis of the streaked data. We checked that, so long as the attenuation effect in the shell is taken into account, the spectroscopic analysis is robust. However, if neglected, the extracted core conditions are sensitive to the selected photon energy interval.

The spectroscopic analysis was then applied to the measured spectra. Figure 9 shows two time-resolved spectra for shot 53259. Early in the deceleration phase $(t=4.38 \mathrm{~ns})$, the $\mathrm{Ly}-\beta$ line is less intense than the $\mathrm{He}-\beta$, and the $\mathrm{He}-\gamma$ is well resolved from the Ly- $\beta$. The electron temperature and density extracted from the analysis are $T_{e}=930( \pm 70) \mathrm{eV}$ and $N_{e}=2.8( \pm 0.4) \times 10^{23} \mathrm{~cm}^{-3}$, respectively. The latter is equivalent to a mass density $\rho=0.9( \pm 0.1) \mathrm{gcm}^{-3}$. Later $(t=4.50 \mathrm{~ns})$, as the core gets hotter and denser, the Ly- $\beta$ intensity increases relative to the $\mathrm{He}-\beta$, and the widths of all the lines are larger because of a stronger Stark broadening effect; in particular, the He- $\gamma$ blends heavily with the Ly- $\beta$ developing a bump-like feature on the low energy side of this line. Also, the bound-free (i.e., radiative recombination) emission becomes important on the high energy side of the Ly- $\beta$ and underneath the Ly- $\gamma$. At this point, in time, the shell attenuation is significant and impacts the analysis
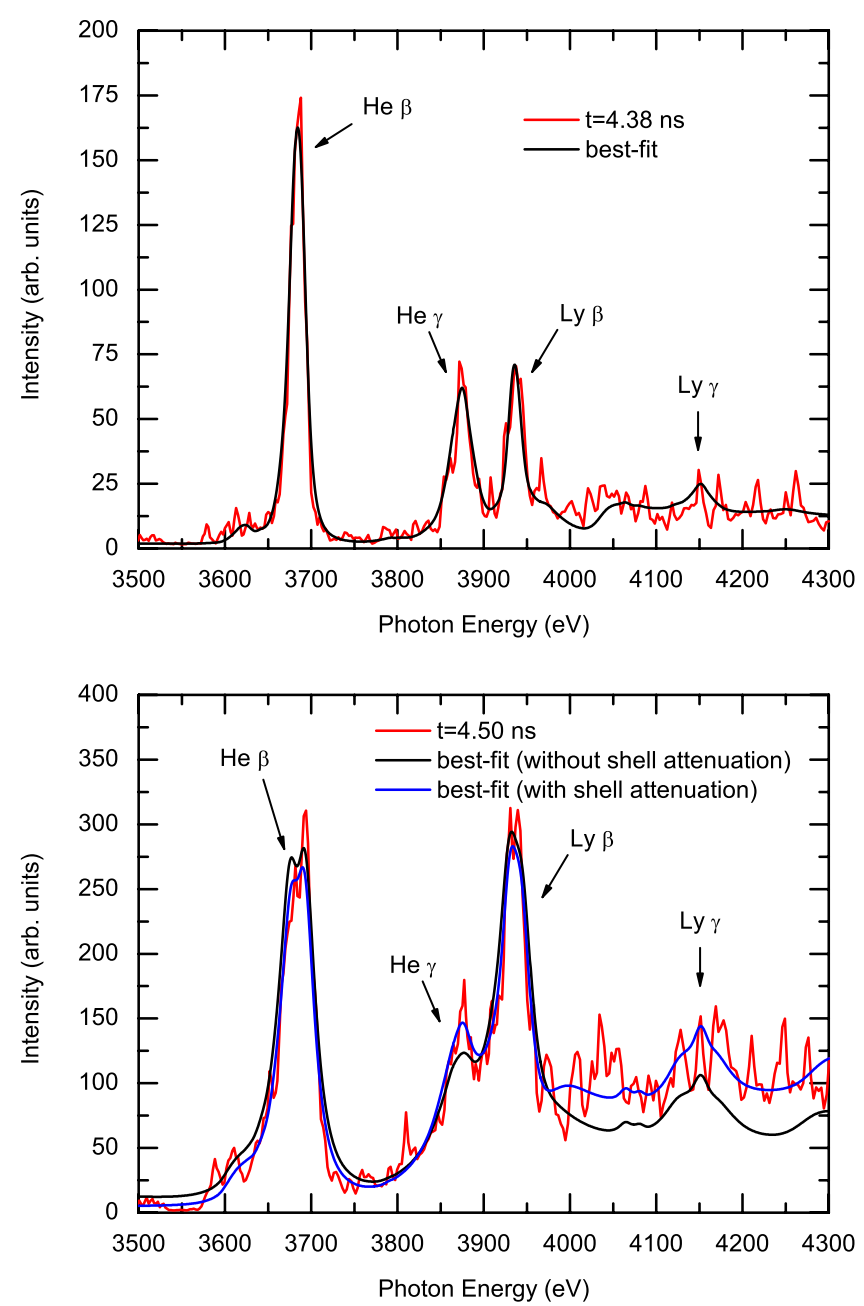

FIG. 9. Spectroscopic model fits of time-resolved spectra from OMEGA shot 53259. Top: result for an early spectrum where the shell attenuation is not important. Bottom: later spectrum and analysis results obtained without and with the shell attenuation effect. 
results. The extracted core conditions not including the shell attenuation effect are $T_{e}=1170 \mathrm{eV}, N_{e}=1.1 \times 10^{24} \mathrm{~cm}^{-3}$, and $\rho=3.8 \mathrm{gcm}^{-3}$, while including this effect, we obtained $T_{e}=1030( \pm 80) \mathrm{eV}, N_{e}=8.9( \pm 1.3) \times 10^{23} \mathrm{~cm}^{-3}$, and $\rho=3.0( \pm 0.4) \mathrm{gcm}^{-3}$. To understand the temperature change, we recall that the $\mathrm{Ly}-\beta$ to $\mathrm{He}-\beta$ intensity ratio is very sensitive to electron temperature and weakly dependent on density. The transmission factor-exponential in Eq. (1) - is a monotonically increasing function of the photon energy. ${ }^{20}$ Thus, the transmission through the shell increases the intensity of the $\mathrm{Ly}-\beta$ relative to the $\mathrm{He}-\beta$ line. Accordingly, not accounting for this effect in the analysis results in an apparent core temperature larger than what it actually is. In a similar way, the larger transmission through the shell of $4000-4300 \mathrm{eV}$ photons enhances the intensity of the Ly- $\gamma$ plus bound-free emissions relative to the $\mathrm{He}-\beta$ line. Keeping in mind that the bound-free emission is proportional to the density, not considering the attenuation effect results in a greater core density. The uncertainties in the extracted values were computed as the standard confidence limits on estimated model parameters, which in turn result from an analysis of the curvature of the weighted- $\chi^{2}$ surface about the minimum, taking into account the correlations between the parameters. $^{71}$ As discussed above, including the attenuation effect is required for extracting reliable values and accordingly uncertainties were only computed when including this effect.

Next, we discuss the time-histories extracted with the spectroscopic method. Fig. 10 displays the time-history of the shell optical thickness averaged over the $3500-4300 \mathrm{eV}$ photon energy range. We emphasize that if the analysis is restricted to the $3500-3975 \mathrm{eV}$ range (only including He- $\beta$, He- $\gamma$ and Ly- $\beta$ ), the optical depth time-history shows an unsettled behavior that renders optical depth values

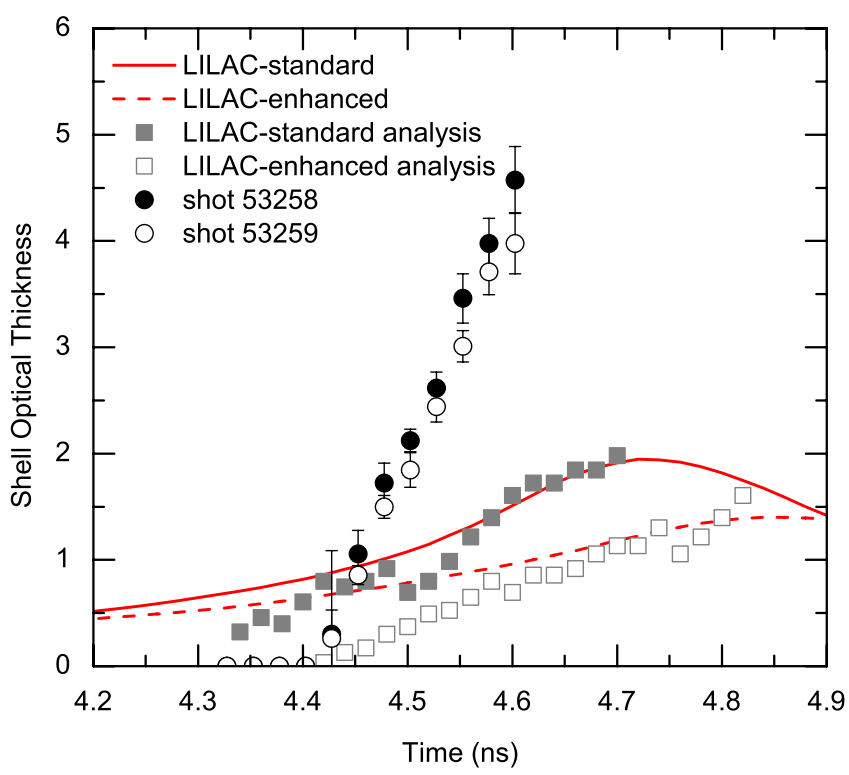

FIG. 10. Time-history of the average shell optical thickness $\left\langle\tau^{s}\right\rangle$ (see text for details) extracted from the analysis of synthetic spectra from LILACstandard and LILAC-enhanced as well as from the analysis of OMEGA shots 53258 and 53259. Predictions of both simulations are shown for comparison. unreliable. This fact confirms that taking into account line and bound-free contributions in the $3500-4300 \mathrm{eV}$ photon energy range is important for a robust analysis. ${ }^{20}$ Predictions from LILAC-standard and LILAC-enhanced results obtained from analysis of synthetic spectra, and those from experimental spectra, are plotted in Fig. 10. Although with a different timing — as already noticed in Fig. 7, simulations show a similar trend in which the average optical depth gradually rises from $\sim 0.5$ to a maximum value of $\sim 1-1.5$. Early in time, the analysis of synthetic spectra underestimates the simulations. This is not surprising since the attenuation effect is still weak so that its footprint in the spectrum is not enough to accurately capture it. Late in time, results from the synthetic analysis slightly overestimate the simulations. This is attributed to differences in the emergent spectra due to the uniformity assumption in the spectroscopic model. In spite of these small deviations, overall, the optical depth timehistories extracted from the analysis of synthetic spectra agree with the simulations. However, a major difference is observed when looking at the experimental case. The onset of the attenuation effect is at approximately $t=4.45 \mathrm{~ns}$, or about $1 \mathrm{~ns}$ after the spike of the laser pulse, then the average optical depth quickly rises to a maximum value of $\sim 4-4.5$. Compared to 1D simulations, this result might suggest that in the experiment, the shell hydrodynamics evolves faster and more abruptly in the final part of the deceleration phase, which also would affect the fuel assembly. A precise answer to this issue would require a time-dependent analysis of the plastic shell temperature, density, and areal-density. We note that the shell optical depth can be written as $\tau_{\nu}^{s}=\mu_{\nu}^{s}\left(\rho^{s} \Delta R\right)$, where $\rho^{s} \Delta R$ is the shell areal-density and $\mu_{\nu}^{s}$ denotes the mass absorption coefficient, which in turn depends on shell temperature and density. As seen, optical depth can be estimated from the spectroscopic analysis, but there is not enough information in the current data to uniquely unfold the time evolution of shell parameters.

Figures 11 and 12 show the time-histories of the core's mass density and electron temperature. In both figures, we include the analysis of the synthetic (from LILAC-standard and LILAC-enhanced) and measured spectra. The former is useful to test the analysis method and see differences between simulations. The latter shows the actual conditions extracted for the experiment. These figures also include lower and upper bounds of the temperature and density radial profiles from the simulations. Since the analysis is timeresolved but space-integrated, we expect that it will produce temperature and density values that fall within these bounds. Figs. 11 and 12 show that this was indeed the case. We also found that if the absorption in the shell is not taken into account, then the analysis of synthetic spectra leads to an artificial monotonically increase of the electron temperature, including an abrupt rising late in the deceleration phase, contrary to what $1 \mathrm{D}$ simulations predict. The shell attenuation effect is, therefore, critical to keep the inferred temperature within bounds and ensure a reliable analysis. This effect was also important for the analysis of measured spectra, and it may reflect the formation of a large areal-density region in the compressed shell that confines the implosion core. As seen in Figs. 11 and 12, both simulations produce 


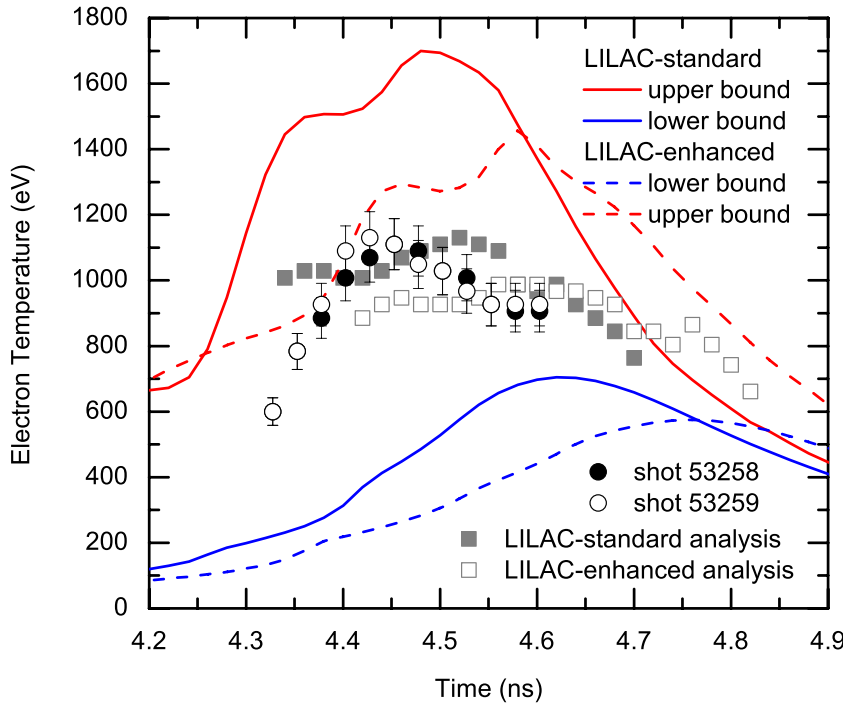

FIG. 11. Time-history of core electron temperature. Results are shown for OMEGA shots 53258 and 53259, and for the analysis of synthetic spectra from LILAC-standard and LILAC-enhanced. Lower and upper bounds predicted by simulations are also plotted for comparison.

temperature and density time-histories that are qualitatively similar, with LILAC-standard progressing a little faster and resulting in a little higher temperature and density values. Timing of LILAC-standard agrees better with the experiment. As the shell decelerates, electron temperature and mass density reach $\sim 1100 \mathrm{eV}$ and $\sim 2.1 \mathrm{gcm}^{-3}$ at $t=4.45 \mathrm{~ns}$. Then, the mass density further increases to $\sim 6.5 \mathrm{gcm}^{-3}$, and the electron temperature drops to $\sim 900 \mathrm{eV}$ at $t=4.6 \mathrm{~ns}$, which is caused by heat conduction to the shell and, mainly, radiative losses, as discussed in Sec. VI. The thermal wave propagating into the shell and the radiation absorption in the shell increase its electron temperature. The measured total radiation power, whose main contribution comes from continuum radiation emitted by the compressed shell, peaks at $t=4.6 \mathrm{~ns}$ (see Fig. 7), after the time of peak core electron temperature, but before stagnation while the density continues to rise. In LILAC-standard, the temperature also peaks at $\sim 1100 \mathrm{eV}(t=4.52 \mathrm{~ns})$ while in LILAC-enhanced, the maximum value is $\sim 1000 \mathrm{eV}(t=4.58 \mathrm{~ns})$. Before this maximum though, the temperature from the synthetic spectra analysis remained roughly constant at $1000 \mathrm{eV}$ and $900 \mathrm{eV}$ for LILAC-standard and LILAC-enhanced, respectively, but in the experiment, it rose quickly from $700 \mathrm{eV}$ to the maximum of $1100 \mathrm{eV}$. After the maximum, in both simulations and experiment, the temperature drops at a comparable rate. This difference in temperature behavior before peak value suggests significant differences between both simulations and experiment in the core heating dynamics driven by the ignitor shock during the deceleration phase. Nonetheless, all density time histories show a similar trend. We note in passing, the consistency between results from the two nominally identical experiments shown in Figs. 11 and 12, i.e., shots 53258 and 53259 . This fact builds confidence on the reproducibility of the results.

So far, we discussed results which are directly extracted from the spectroscopic analysis. However, the extracted

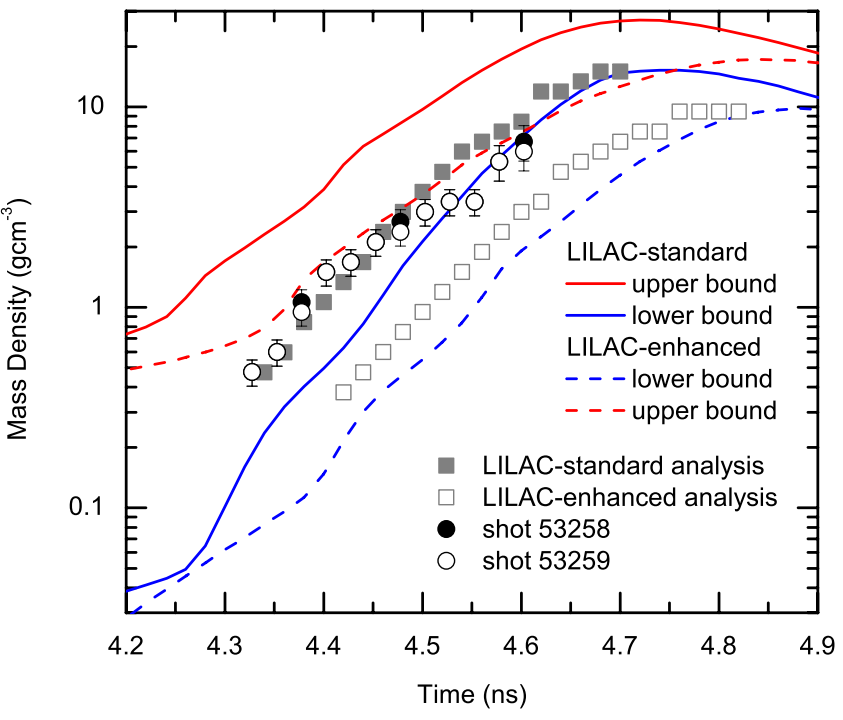

FIG. 12. Time-history of core mass density. Results are shown for OMEGA shots 53258 and 53259, and for the analysis of synthetic spectra from LILAC-standard and LILAC-enhanced. Lower and upper bounds predicted by simulations are also plotted for comparison.

temperature and density can be used to examine the time evolution of other quantities. Within the uniform model assumption, an effective core radius $R$ can be calculated from $R=\left(3 \delta m_{0} / 4 \pi \rho\right)^{1 / 3}$, where $\rho$ is the mass density extracted from the analysis, $m_{0}$ is the total (initial) core mass, and $\delta$ represents the fraction of the mass effectively contributing to the formation of the emission spectrum. Hence, $R$ actually denotes the radius of the effective core volume that significantly contributes to the spectrum. There is not enough information in the data to experimentally constrain and determine the fraction $\delta$, so we initially assume $\delta=1$. Accordingly, Fig. 13 shows the resulting core radius time-histories. Values predicted by LILAC-standard and LILAC-enhanced are also

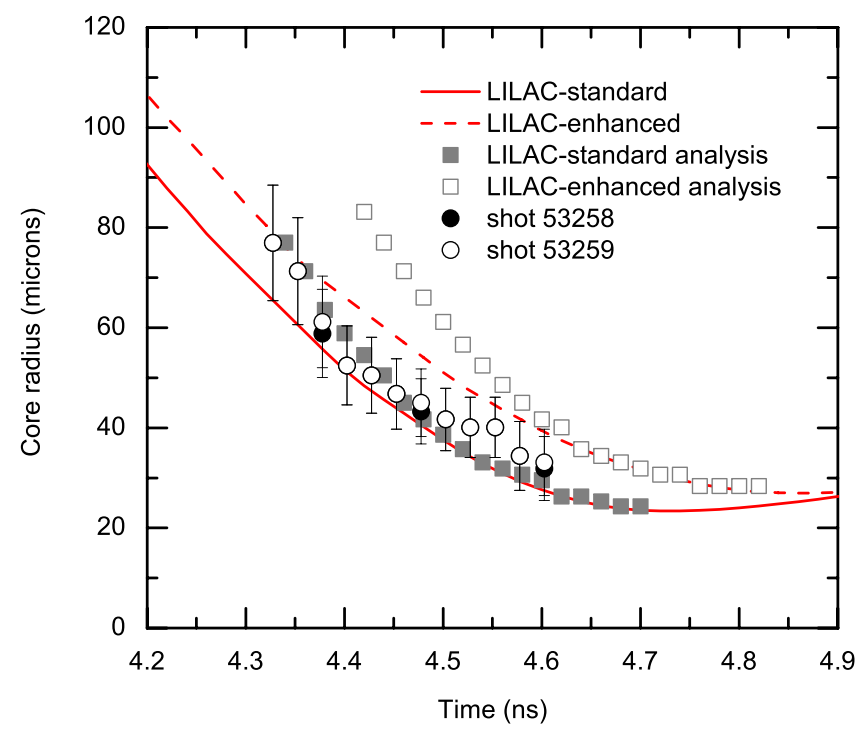

FIG. 13. Time-history of extracted core radius. We plot the results for OMEGA shots 53258 and 53259 and for synthetic spectra from LILACstandard and LILAC-enhanced. Core radii predicted by both types of simulations are also shown for comparison. 
plotted for comparison. The analysis of synthetic spectra predicts, early in time, a core radius greater than that of the simulation (either LILAC-standard or LILAC-enhanced). As time progresses, better agreement between analysis and simulations is observed. Keeping in mind that the extracted temperature and density can be interpreted as intensity-averaged values, ${ }^{72}$ differences can be explained as follows. The $1 \mathrm{D}$ simulation temperature radial profiles indicate that, late in time, nearly all the core is hot enough to radiate appreciably, the extracted $\rho$ is then representative of the entire core volume, $\delta=1$ becomes a good approximation and, therefore, the core radius $R$ inferred from the analysis compares well with simulation prediction. However, early in time, only the inner central portion of the core achieves temperatures high enough to light up the Ar K-shell lines. According to simulation, density increases from the inside out, and, consistently, the analysis leads to a density value closer to the LILAC lower bound. Then, substituting the extracted density in the formula above, with the assumption $\delta=1$, yields a core radius greater than that of the simulations. Considering that only the central region of the core volume significantly contributes to the emission spectrum, the opposite would be expected. Based on this comparison between the results from LILAC and those from the analysis of synthetic spectra, we can then obtain a better estimate for the fraction $\delta$ of fuel mass contributing to the formation of the emergent spectra. An upper bound for this fraction is calculated as $\delta_{\max }=4 \pi R_{\text {sim }}^{3} \rho / 3 m_{0}$, with $R_{\text {sim }}$ being the core radius predicted by $1 \mathrm{D}$ hydrodynamic simulations. For both LILACstandard and LILAC-enhanced, we found that, starting from $\delta_{\max } \approx 0.5, \delta_{\max }$ increases at a constant rate of $d \delta_{\max } / d t \approx$ $0.0023 \mathrm{ps}^{-1}$ while core temperature is increasing. After temperature peaks, $\delta_{\max } \approx 1$.

The pressure inside the core can be calculated with an ideal gas equation of state (EOS) for Ar and D atoms which is a reasonable approximation considering the core temperature and density conditions. Figure 14 displays the total core pressure, i.e., including the contributions of deuterium and $\mathrm{Ar}$ ions and electrons, taking into account the shell attenuation effect. Not including this effect results in significantly larger pressure values. The pressure calculation assumed that ions and electrons have the same temperature. This approximation is reasonable since for the temperature and density ranges of the analysis the deuterium ions and electrons (whose contributions dominate the total pressure) have thermalization times in the range from $4 \mathrm{ps}$ to $17 \mathrm{ps}$, and the analysis covers a time interval of $330 \mathrm{ps.} \mathrm{Moreover,}$ each of the time-resolved spectra used for the analysis was integrated over a $75 \mathrm{ps}$ time sub-interval, i.e., about four times larger than the longest thermalization time. Figure 14 also shows the local pressure at the core boundary predicted by LILAC-standard and LILAC-enhanced. The comparison between these values and those from the analysis is useful for an interpretation of $P d V$-work calculation shown in Sec. VI. The pressure calculation from LILACstandard compares better to the experiment than that from LILAC-enhanced, but towards the end of the observations, the experiment tends to have less pressure than the simulation.

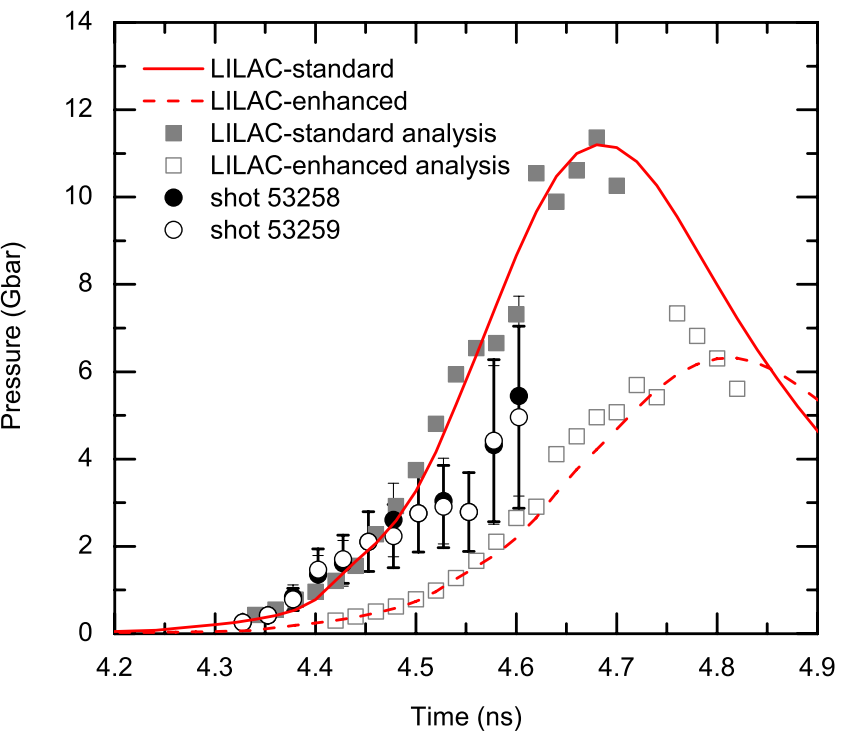

FIG. 14. Time-history of extracted core pressure. We plot the results for OMEGA shots 53258 and 53259 and for synthetic spectra from LILACstandard and LILAC-enhanced. Core pressure at the boundary predicted by both types of simulations are also shown for comparison.

\section{ENERGY BALANCE ANALYSIS}

Based on the pressure and density, the time-histories, a plot of the core's pressure vs. density suggests, an interpretation of the deceleration phase in terms of a thermodynamic polytropic process characterized by the equation $P \rho^{-\gamma}=$ constant, where $\gamma$ denotes the polytropic exponent. Figure 15 shows the pressure-density diagram in a log-log plot. During the analysis time-interval, the density increases monotonically while the temperature, first, increases and then decreases. Accordingly, fits to extract the polytropic exponent were performed for both temperature regimes. Table I displays the values of $\gamma$ for simulations and experiment. The adiabatic case would be described by $\gamma=\frac{5}{3} \approx 1.67$ (considering an ideal monoatomic gas). A deviation from the adiabatic case is expected since the core exchanges energy with the surrounding shell through thermal and radiation transport. The energy loss due to nuclear fusion reactions was negligible in these implosions. The $\gamma$ 's extracted for LILAC-standard and LILAC-enhanced simulations are comparable, with $\gamma$ for LILAC-enhanced being slightly higher during the temperature drop. For the first time-interval of the analysis, the polytropic fits suggest a nearly isothermal implosion, i.e., $\gamma \approx 1$, which is consistent with the modest increase in temperature observed in Fig. 11 before it peaks. Results for shots 53258 and 53259 compare well with each other. However, the comparison between the simulations and experiment indicates that during the first stage of the deceleration phase, the experiment is closer to an adiabatic compression. While the temperature decreases, simulations and experiment show a similar (within uncertainties) behavior.

In order to gain more insight, we consider the energy conservation statement for the implosion core. A formal integration of the fluid equations for the core leads to the following result: 

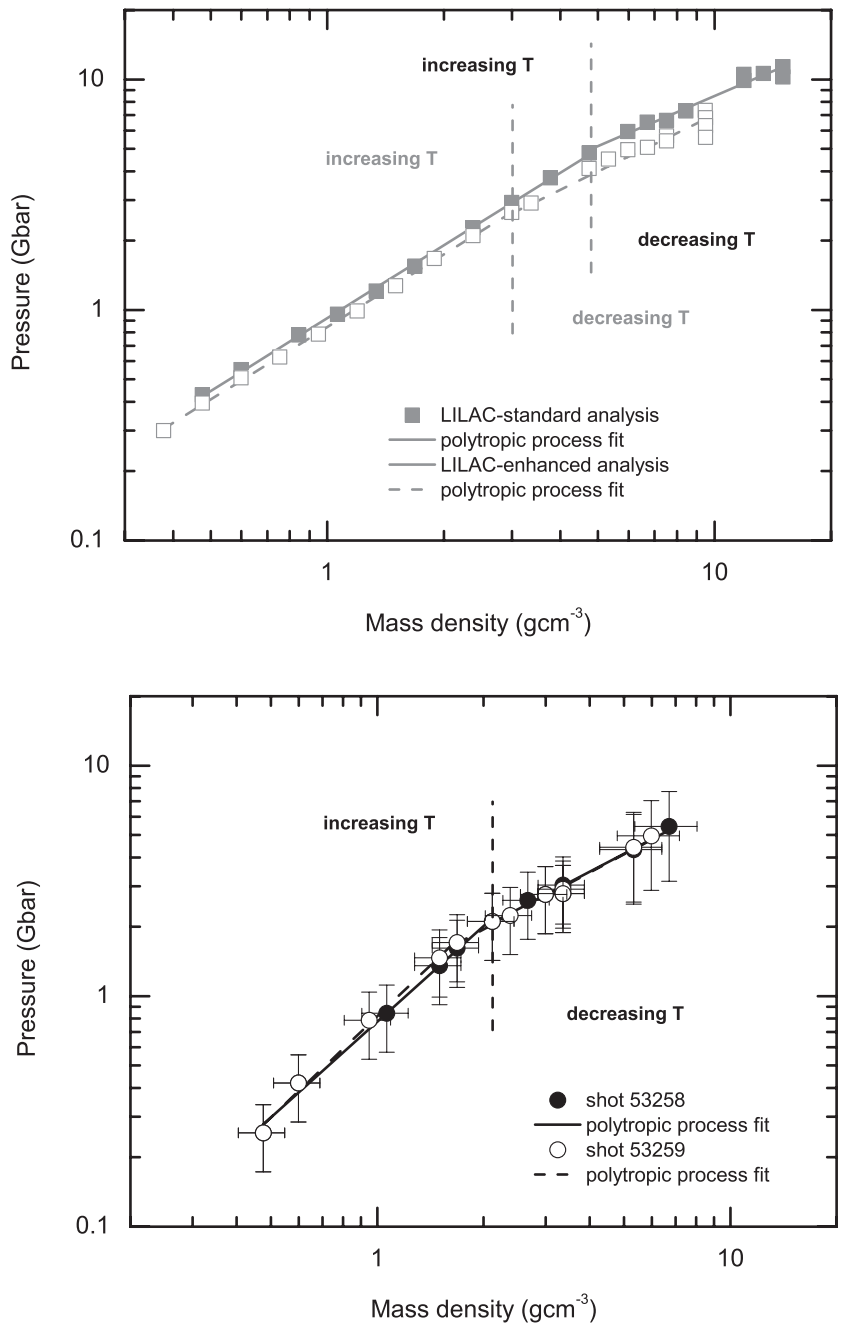

FIG. 15. P- $\rho$ diagram of the imploded core extracted from the spectroscopic analysis of both synthetic (top) and experimental (bottom) spectra. Linear fits to the data are shown from comparison with the adiabatic case.

$$
\frac{d E}{d t}=\frac{d}{d t}(U+K)=\dot{W}+\dot{F}+\dot{J}
$$

where $E=U+K$ is the total energy of the core, $U$ is the total internal energy, $K$ the total kinetic energy, $\dot{W}$ the rate of work done by the shell on the core, $\dot{F}$ the rate of thermal transport through the surface of the core, and $\dot{J}$ the power radiated by the core. Net changes in $W, F$, and $J$ can be obtained from LILAC-standard and LILAC-enhanced simulation's output. For both simulations and experiment, they can also be computed using the time-histories of temperature, density, radius (volume), and pressure determined from the spectroscopic analysis (Figs. 11-14) and the formulas

TABLE I. Polytropic exponent $\gamma$ for simulations and experiment determined by least-square fits to the data shown in Fig. 15. In the experimental case, $\gamma$ and $\Delta \gamma$ take into account uncertainties in mass density and pressure.

\begin{tabular}{lccccc}
\hline \hline \multirow{2}{*}{ Polytropic exponent $\gamma$} & \multicolumn{2}{c}{ LILAC simulation } & & \multicolumn{2}{c}{ Experiment } \\
\cline { 2 - 3 } \cline { 5 - 6 } \cline { 5 - 6 } & Standard & Enhanced & & Shot 53258 & Shot 53259 \\
\hline 1st stage: increasing $T$ & 1.05 & 1.05 & & $1.4 \pm 0.3$ & $1.5 \pm 0.4$ \\
2nd stage: decreasing $T$ & 0.72 & 0.82 & & $0.8 \pm 0.4$ & $0.8 \pm 0.3$ \\
\hline \hline
\end{tabular}

from Ref. 73 and 74. In order to compare the results for shots 53258 and 53259 with those extracted from the analysis of synthetic spectra, net changes were computed over equivalent time-intervals relative to the time of maximum temperature, i.e., counted before and after the temperature peak.

In this connection, we found that, when using the core radius obtained from the mass conservation assumption (i.e., $\delta=1), P d V$-work computed from the analysis of synthetic spectra overestimates what simulations predict particularly for the time sub-interval before the maximum temperature. This indicates that a better estimate for the core size is needed for a meaningful calculation of the compression work and connects with the idea that during the first stage of the deceleration phase only a fraction of the core mass contributes to the formation of emission spectrum. In fact, if we consider the temperature, density, and pressure values from the analysis of synthetic spectra, but use the actual core radius, $R_{\text {sim }}$, predicted by the simulation (or, equivalently, $\delta=\delta_{\max }$ ), a significant improvement in the calculation of compression work was observed. Changes in $F$ and $J$ were small because dependence on core radius (volume) and compression rate is much weaker than that of $W$. From now on, therefore, we will refer to the results using $\delta=\delta_{\max }$ to estimate the core volume. In the case of the synthetic spectra analysis, by definition, this leads to $R=R_{\text {sim }}$. In the experiment, $\delta_{\max }$ is determined according to the behavior discussed in Sec. V.

Table II shows the results for the entire time-interval of the analysis as well as the breakdown associated with the time sub-intervals of increasing and decreasing temperatures. The negative sign indicates a net loss of energy, and the positive $W$ represents compression work done on the core. Overall, it is seen that in the simulations and experiment, the loss of energy is larger while the temperature is decreasing, and it is dominated by the radiated power. Differences between simulations can be observed when looking at the results for each time subinterval; however, over the entire interval of analysis, in both simulations energy losses due to radiation and thermal transport represent $\sim 75 \%-80 \%$ of compression work, thus resulting in a net increase in total energy. Calculations of $W$ and $F$ obtained from the analysis of synthetic spectra agree reasonably well with the values of LILAC simulations. However, radiative losses are underestimated. Main contribution to total radiative losses (i.e., over the entire photon-energy range) comes from bremsstrahlung emission of free electrons from D and Ar ions. Discrepancies can be attributed to the fact that the spectroscopic analysis relies on the Ar K-shell emission and, therefore, extracted conditions are more representative of the central portion of the core, which is hotter but not as dense as the outer region. Considering that bremsstrahlung emission scales with density as $\rho^{2}$, an underestimation in $J$ can actually be expected. For OMEGA shots 53258 and 53259, the good consistency found in the time-resolved analysis leads to very similar results in the core energy balance. Compression work values are closer to those predicted by LILAC-standard, although in the interval of decreasing temperature work done by the shell on the core is greater in the experimental case. Thermal conduction contribution is comparable to what simulations 
TABLE II. Breakdown of contributions to the change in total energy $E$ of the core. $W$ is the compression work done on the core, and $F$ and $J$ denote contributions due to thermal and radiation transport, respectively. Note that $\Delta E=\Delta W+\Delta F+\Delta J$. Only results using $\delta=\delta_{\max }$ are shown (see text for details).

\begin{tabular}{|c|c|c|c|c|c|c|c|}
\hline \multicolumn{2}{|c|}{ Energy terms $(J)$} & \multicolumn{2}{|c|}{ LILAC simulation } & \multicolumn{2}{|c|}{ Synthetic spectra analysis } & \multicolumn{2}{|c|}{ Experiment } \\
\hline & & Standard & Enhanced & Standard & Enhanced & Shot 53258 & Shot 53259 \\
\hline & $\Delta W$ & 77.05 & 46.11 & 83.86 & 50.06 & 73.50 & 68.37 \\
\hline 1st stage & $\Delta F$ & -4.35 & -2.32 & -10.97 & -7.93 & -9.01 & -7.61 \\
\hline \multirow[t]{3}{*}{ Increasing $T$} & $\Delta J$ & -28.21 & -18.09 & -16.11 & -8.21 & -8.16 & -5.64 \\
\hline & $\Delta E$ & 44.49 & 25.70 & 56.78 & 33.92 & 56.33 & 55.12 \\
\hline & $\Delta W$ & 84.38 & 55.13 & 84.75 & 63.41 & 92.89 & 104.92 \\
\hline 2nd stage & $\Delta F$ & -10.88 & -5.74 & -9.48 & -7.50 & -11.26 & -13.95 \\
\hline \multirow[t]{3}{*}{ Decreasing $T$} & $\Delta J$ & -89.65 & -49.94 & -73.66 & -46.53 & -32.57 & -34.56 \\
\hline & $\Delta E$ & -16.15 & -0.55 & 1.61 & 9.38 & 49.06 & 56.41 \\
\hline & $\Delta W$ & 161.43 & 101.24 & 168.61 & 113.47 & 166.39 & 173.29 \\
\hline Entire & $\Delta F$ & -15.23 & -8.06 & -20.45 & -15.43 & -20.27 & -21.56 \\
\hline \multirow[t]{2}{*}{ Time interval } & $\Delta J$ & -117.86 & -68.03 & -89.77 & -54.74 & -40.73 & -40.20 \\
\hline & $\Delta E$ & 28.34 & 25.15 & 58.39 & 43.30 & 105.39 & 111.53 \\
\hline
\end{tabular}

predict. Major differences between experiment and simulation are found in the radiative losses. In this case, $J$ values are better described by LILAC-enhanced; however, as observed in the results for the entire time interval, radiative losses are appreciably smaller in the experiment than in the simulation, which leads to a net increment to the total core energy about four times larger. This result must be taken with caution though, since as discussed above radiative losses are likely underestimated. However, still assuming an underestimation of a factor of two, the net change of the total core energy in the experiment would duplicate that predicted by the simulation. In this regard, for the time interval in the deceleration phase in which the Ar emission signal is bright enough for a spectroscopic diagnosis, the energy balance analysis indicates that the compression work done by the shell on the core overcomes the energy losses by thermal conduction and radiation transfer which results in an increase of core total energy greater than what LILAC simulations predict.

Finally, assuming a monoatomic ideal gas equation of state and the polytropic process equation, a further elaboration of Eq. (3)—see the Appendix —shows that, if $\gamma<\frac{5}{3}$, in a compression the net energy exchange (by thermal and/or radiation transport) is less than the change in kinetic energy. Moreover, in the case of an imploding core in the deceleration phase, the change in kinetic energy is negative, and thus, $\gamma<\frac{5}{3}$ has a clear interpretation: there is a net energy transfer from the core to the compressed shell. As seen in Tables I and II, this is always the case in the analysis discussed here.

\section{CONCLUSIONS}

In this work, the observation with a streaked crystal spectrometer of the spectroscopic signature of an Ar tracer added to the deuterium gas fill of the target was used for the first time-resolved diagnosis of core conditions in shockignition experiments at OMEGA. The extracted temperature and density time-histories are representative of the state of the core during the implosion deceleration and fuel burning phases. The data analysis confirms the importance of the shell attenuation effect in thick-wall targets previously noted on time-integrated spectroscopic measurements. ${ }^{20}$ This effect must be taken into account in the spectroscopic analysis in order to obtain reliable results.

Two types of calculations using the 1D hydrodynamic code LILAC were performed in order to simulate the experiments: (1) LILAC-standard which did not take into account the effect of CBET, and a flux-limiting factor $f=0.06$ was used in the thermal transport model, and (2) LILACenhanced which considered non-local thermal transport as well as the CBET effect. Time-history of temperature and density profiles predicted by both simulations were postprocessed to obtain the space integrated but time-resolved $\mathrm{Ar}$ spectra. The collection of synthetic spectra was analyzed with the same spectroscopic model used for the experimental case. Overall, results extracted from LILAC-standard synthetic spectra compare better with measurements; however, neither of the simulations approximates well all experimental observations. In particular, discrepancies in temperature behavior before the peak value between both simulations and experiment suggest a significant difference in the core heating dynamics driven by the ignitor shock during the deceleration phase. Also, a fast increase of the shell's average optical depth to values $\sim 4$ is observed in the experiment after the maximum in temperature. However, the analysis of synthetic spectra revealed that in the simulations, the shell's optical depth rises at a lower pace and remains under $\sim 2$ during the time-interval of analysis. This fact may suggest differences in the dynamics of fuel assembly between 1D simulations and experiment. A deeper look into these issues would probably require time-dependent diagnosis of shell conditions, 2D hydrodynamic simulations and an assessment of mixing effects. Such study is beyond the scope of this work, but it might be the focus of a future experimental campaign using core- and shell-doped targets.

Finally, the value of the spectroscopic diagnosis is further demonstrated by exploiting the results of the timeresolved analysis to carry out a thermodynamic and energy balance study of the core compression. A plot of the core 
pressure vs. density leads to an interpretation of the deceleration phase in terms of a two-stage polytropic process. Also, the work done by the compressed shell on the core as well as core energy losses due to thermal and radiation transport were computed, which allowed us to study the energy balance of the implosion core during the deceleration phase. In this regard, the results point out to an increase in the total energy of the core greater than what LILAC simulations predict. The time-dependent nature of the measurements and the detailed information provided by the spectroscopic method discussed here open up a new window into the dynamics of ICF implosion cores and contributes to advance the field of the high energy density physics.

\section{ACKNOWLEDGMENTS}

This work was supported by DOE/NLUF Grants DEFG52-09NA29042 and DE-NA0000859, and LLNL. R. Florido has also been supported by Spanish Ministry of Science and Innovation Grant No. ENE2009-11208 and by the EURATOM within the ToIFE activities. We thank V. Glebov for his help with the processing of the NTD data.

\section{APPENDIX: CORE-SHELL ENERGY TRANSFER}

From Eq. (3) and for a differential time interval $d t$, the energy conservation becomes

$$
d U+d K=d W+d Q
$$

where $d Q=d F+d J$. Assuming a monoatomic ideal gas, i.e., $P V=n R T$ and $U=\frac{3}{2} n R T$ (where $n$ is the number of moles and $R$ is the ideal gas constant), and considering a thermodynamic polytropic process, i.e., $P V^{\gamma}=A=$ constant $(A>0)$, the change in internal energy can be written as

$$
d U=\frac{3}{2} A(1-\gamma) V^{-\gamma} d V,
$$

and the work $d W=-P d V$ done by the shell is given by

$$
d W=-A V^{-\gamma} d V .
$$

By substituting Eqs. (A2) and (A3) into Eq. (A1), it follows

$$
d Q-d K=\frac{3}{2} A\left(\frac{5}{3}-\gamma\right) V^{-\gamma} d V .
$$

Throughout the deceleration phase, the core is being compressed, i.e., $d V<0$, and is also slowing down, i.e., $d K<0$. Therefore, if $\gamma<\frac{5}{3}$, from Eq. (A4), it is found that during the deceleration phase $d Q<d K<0$. A negative value for $d Q$ indicates that there is a net energy transfer from the core to the compressed shell due to thermal and radiation transport.

${ }^{1}$ R. Betti, C. D. Zhou, K. S. Anderson, L. J. Perkins, W. Theobald, and A. A. Solodov, Phys. Rev. Lett. 98, 155001 (2007).

${ }^{2}$ W. Theobald, R. Betti, C. Stoeckl, K. S. Anderson, J. A. Delettrez, V. Y. Glebov, V. N. Goncharov, F. J. Marshall, D. N. Maywar, R. L. McCrory, D. D. Meyerhofer, P. B. Radha, T. C. Sangster, W. Seka, D. Shvarts, V. A. Smalyuk, A. A. Solodov, B. Yaakobi, C. D. Zhou, J. A. Frenje, C. K. Li, F. H. Seguin, R. D. Petrasso, and L. J. Perkins, Phys. Plasmas 15, 056306 (2008).
${ }^{3}$ L. J. Perkins, R. Betti, K. N. LaFortune, and W. H. Williams, Phys. Rev. Lett. 103, 045004 (2009).

${ }^{4}$ B. Canaud and M. Temporal, New J. Phys. 12, 043037 (2010).

${ }^{5}$ X. Ribeyre, G. Schurtz, M. Lafon, S. Galera, and S. Weber, Plasma Phys. Controlled Fusion 51, 015013 (2009).

${ }^{6}$ X. Ribeyre, M. Lafon, G. Schurtz, M. Olazabal-Loumé, J. Breil, S. Galera, and S. Weber, Plasma Phys. Controlled Fusion 51, 124030 (2009).

${ }^{7}$ M. Lafon, X. Ribeyre, and G. Schurtz, Phys. Plasmas 17, 052704 (2010).

${ }^{8}$ S. Atzeni, A. Schiavi, and A. Marocchino, Plasma Phys. Controlled Fusion 53, 035010 (2011).

${ }^{9}$ R. Nora and R. Betti, Phys. Plasmas 18, 082710 (2011).

${ }^{10} \mathrm{X}$. Ribeyre, V. T. Tikhonchuk, J. Breil, M. Lafon, and E. Le Bel, Phys. Plasmas 18, 102702 (2011).

${ }^{11}$ M. R. Terry, L. J. Perkins, and S. M. Sepke, Phys. Plasmas 19, 112705 (2012).

${ }^{12}$ S. Atzeni, A. Marocchino, A. Schiavi, and G. Schurtz, New J. Phys. 15, 045004 (2013)

${ }^{13}$ C. J. Davie and R. G. Evans, Phys. Rev. Lett. 110, 185002 (2013).

${ }^{14}$ R. Florido, R. C. Mancini, T. Nagayama, R. Tommasini, J. A. Delettrez, S. P. Regan, and B. Yaakobi, Rev. Sci. Instrum. 81, 10E307 (2010).

${ }^{15}$ A. R. Bell and M. Tzoufras, Plasma Phys. Controlled Fusion 53, 045010 (2011).

${ }^{16}$ S. Depierreux, C. Goyon, K. Lewis, H. Bandulet, D. T. Michel, G. Loisel, V. Yahia, V. Tassin, C. Stenz, N. G. Borisenko, W. Nazarov, J. Limpouch, P. E. Masson Laborde, P. Loiseau, M. Casanova, S. Nicolaï, Ph. Hüller, D. Pesme, C. Riconda, V. T. Tikhonchuk, and C. Labaune, Plasma Phys. Controlled Fusion 53, 124034 (2011).

${ }^{17}$ S. Atzeni, A. Marocchino, and A. Schiavi, Phys. Plasmas 19, 090702 (2012).

${ }^{18}$ K. S. Anderson, R. Betti, P. W. McKenty, T. J. B. Collins, M. Hohenberger, W. Theobald, R. S. Craxton, J. A. Delettrez, M. Lafon, J. A. Marozas, R. Nora, S. Skupsky, and A. Shvydky, Phys. Plasmas 20, 056312 (2013).

${ }^{19}$ W. Theobald, R. Nora, M. Lafon, A. Casner, X. Ribeyre, K. S. Anderson, R. Betti, J. A. Delettrez, J. A. Frenje, V. Y. Glebov, O. V. Gotchev, M. Hohenberger, S. X. Xu, F. J. Marshall, D. D. Meyerhofer, T. C. Sangster, G. Schurtz, W. Seka, V. A. Smalyuk, C. Stoeckl, and B. Yaakobi, Phys. Plasmas 19, 102706 (2012).

${ }^{20}$ R. Florido, R. C. Mancini, T. Nagayama, R. Tommasini, J. A. Delettrez, S. P. Regan, and B. Yaakobi, Phys. Rev. E 83, 066408 (2011).

${ }^{21}$ S. D. Baton, M. Koenig, E. Brambrink, H. P. Schlenvoigt, C. Rousseaux, G. Debras, S. Laffite, P. Loiseau, F. Philippe, X. Ribeyre, and G. Schurtz, Phys. Rev. Lett. 108, 195002 (2012).

${ }^{22}$ C. Goyon, S. Depierreux, V. Yahia, G. Loisel, C. Baccou, C. Courvoisier, N. G. Borisenko, A. Orekhov, O. Rosmej, and C. Labaune, Phys. Rev. Lett. 111, 235006 (2013).

${ }^{23}$ W. Shang, H. Wei, Z. Li, R. Yi, T. Zhu, T. Song, C. Huang, and J. Yang, Phys. Plasmas 20, 102702 (2013).

${ }^{24}$ M. Hohenberger, W. Theobald, S. X. Hu, K. S. Anderson, R. Betti, T. R. Boehly, A. Casner, D. E. Fratanduono, M. Lafon, D. D. Meyerhofer, R. Nora, X. Ribeyre, T. C. Sangster, G. Schurtz, W. Seka, C. Stoeckl, and B. Yaakobi, Phys. Plasmas 21, 022702 (2014).

${ }^{25}$ S. Atzeni, X. Ribeyre, G. Schurtz, A. J. Schmitt, B. Canaud, R. Betti, and L. J. Perkins, Nucl. Fusion 54, 054008 (2014).

${ }^{26}$ D. Batani, S. Baton, A. Casner, S. Depierreux, M. Hohenberger, O. Klimo, M. Koenig, C. Labaune, X. Ribeyre, C. Rousseaux, G. Schurtz, W. Theobald, and V. T. Tikhonchuk, Nucl. Fusion 54, 054009 (2014).

${ }^{27}$ S. P. Regan, B. Yaakobi, T. R. Boehly, R. Epstein, J. Delettrez, V. Y. Glebov, V. N. Goncharov, P. A. Jaanimagi, J. P. Knauer, F. J. Marshall, R. L. McCrory, D. D. Meyerhofer, P. B. Radha, T. C. Sangster, V. A. Smalyuk, J. Soures, C. Stoeckl, R. C. Mancini, D. A. Haynes, Jr., L. Welser-Sherrill, J. A. Koch, R. Tommasini, and H. Sawada, High Energy Density Phys. 5, 234 (2009).

${ }^{28}$ B. Yaakobi, I. Pelah, and J. Hoose, Phys. Rev. Lett. 37, 836 (1976).

${ }^{29}$ B. Yaakobi, C. Stoeckl, W. Seka, J. A. Delettrez, T. C. Sangster, and D. D. Meyerhofer, Phys. Plasmas 12, 062703 (2005).

${ }^{30}$ B. Yaakobi, D. Steel, E. Thorsos, A. Hauer, and B. Perry, Phys. Rev. Lett. 39, 1526 (1977).

${ }^{31}$ B. Yaakobi, S. Skupsky, R. L. McCrory, C. F. Hooper, H. Deckman, P. Bourke, and J. M. Soures, Phys. Rev. Lett. 44, 1072 (1980).

${ }^{32}$ C. J. Keane, B. A. Hammel, A. L. Osterheld, and D. R. Kania, Phys. Rev. Lett. 72, 3029 (1994).

${ }^{33}$ H. Nishimura, T. Kiso, H. Shiraga, T. Endo, K. Fujita, A. Sunahara, H. Takabe, Y. Kato, and S. Nakai, Phys. Plasmas 2, 2063 (1995). 
${ }^{34}$ C. F. Hooper, Jr., D. P. Kilcrease, R. C. Mancini, L. A. Woltz, D. K. Bradley, P. A. Jaanimagi, and M. C. Richardson, Phys. Rev. Lett. 63, 267 (1989).

${ }^{35}$ D. A. Haynes, Jr., D. T. Garber, C. F. Hooper, Jr., R. C. Mancini, Y. T. Lee, D. K. Bradley, J. Delettrez, R. Epstein, and P. A. Jaanimagi, Phys. Rev. E 53, 1042 (1996).

${ }^{36}$ N. C. Woolsey, B. A. Hammel, C. J. Keane, A. Asfaw, C. A. Back, J. C. Moreno, J. K. Nash, A. Calisti, C. Mosse, R. Stamm, B. Talin, L. Klein, and R. W. Lee, Phys. Rev. E 56, 2314 (1997).

${ }^{37}$ S. P. Regan, J. A. Delettrez, R. Epstein, P. A. Jaanimagi, B. Yaakobi, V. A. Smalyuk, F. J. Marshall, D. D. Meyerhofer, and W. Seka, Phys. Plasmas 9, 1357 (2002).

${ }^{38}$ R. Florido, T. Nagayama, R. C. Mancini, R. Tommasini, J. A. Delettrez, S. P. Regan, V. A. Smalyuk, R. Rodríguez, and J. M. Gil, Rev. Sci. Instrum. 79, 10E310 (2008).

${ }^{39}$ I. Golovkin, R. Mancini, S. Louis, Y. Ochi, K. Fujita, H. Nishimura, H. Shirga, N. Miyanaga, H. Azechi, R. Butzbach, I. Uschmann, E. Förster, J. Delettrez, J. Koch, R. W. Lee, and L. Klein, Phys. Rev. Lett. 88, 045002 (2002).

${ }^{40}$ L. Welser-Sherrill, R. C. Mancini, J. A. Koch, N. Izumi, R. Tommasini, S. W. Haan, D. A. Haynes, I. E. Golovkin, J. J. MacFarlane, J. A. Delettrez, F. J. Marshall, S. P. Regan, V. A. Smalyuk, and G. Kyrala, Phys. Rev. E 76, 056403 (2007).

${ }^{41}$ T. Nagayama, R. C. Mancini, R. Florido, R. Tommasini, J. A. Koch, J. A. Delettrez, S. P. Regan, V. A. Smalyuk, L. A. Welser-Sherrill, and I. E. Golovkin, Rev. Sci. Instrum. 79, 10E921 (2008).

${ }^{42}$ T. Nagayama, R. C. Mancini, R. Florido, D. Mayes, R. Tommasini, J. A. Koch, J. A. Delettrez, S. P. Regan, and V. A. Smalyuk, Phys. Plasmas 21, 050702 (2014).

${ }^{43}$ T. Nagayama, R. C. Mancini, R. Florido, D. Mayes, R. Tommasini, J. A. Koch, J. A. Delettrez, S. P. Regan, and V. A. Smalyuk, Phys. Plasmas 19, 082705 (2012).

${ }^{44}$ National Nuclear Security Administration's Path Forward to Achieving Ignition in the Inertial Confinement Fusion Program, Tech. Rep. (U. S. Department of Energy, 2012).

${ }^{45}$ M. J. Edwards, P. K. Patel, J. D. Lindl, L. J. Atherton, S. H. Glenzer, S. W. Haan, J. D. Kilkenny, O. L. Landen, E. I. Moses, A. Nikroo, R. Petrasso, T. C. Sangster, P. T. Springer, S. Batha, R. Benedetti, L. Bernstein, R. Betti, D. L. Bleuel, T. R. Boehly, D. K. Bradley, J. A. Caggiano, D. A. Callahan, P. M. Celliers, C. J. Cerjan, K. C. Chen, D. S. Clark, G. W. Collins, E. L. Dewald, L. Divol, S. Dixit, T. Doeppner, D. H. Edgell, J. E. Fair, M. Farrell, R. J. Fortner, J. Frenje, M. G. G. Johnson, E. Giraldez, V. Y. Glebov, G. Grim, B. A. Hammel, A. V. Hamza, D. R. Harding, S. P. Hatchett, N. Hein, H. W. Herrmann, D. Hicks, D. E. Hinkel, M. Hoppe, W. W. Hsing, N. Izumi, B. Jacoby, O. S. Jones, D. Kalantar, R. Kauffman, J. L. Kline, J. P. Knauer, J. A. Koch, B. J. Kozioziemski, G. Kyrala, K. N. LaFortune, S. L. Pape, R. J. Leeper, R. Lerche, T. Ma, B. J. MacGowan, A. J. MacKinnon, A. Macphee, E. R. Mapoles, M. M. Marinak, M. Mauldin, P. W. McKenty, M. Meezan, P. A. Michel, J. Milovich, J. D. Moody, M. Moran, D. H. Munro, C. L. Olson, K. Opachich, A. E. Pak, T. Parham, H.-S. Park, J. E. Ralph, S. P. Regan, B. Remington, H. Rinderknecht, H. F. Robey, M. Rosen, S. Ross, J. D. Salmonson, J. Sater, D. H. Schneider, F. H. Seguin, S. M. Sepke, D. A. Shaughnessy, V. A. Smalyuk, B. K. Spears, C. Stoeckl, W. Stoeffl, L. Suter, C. A. Thomas, R. Tommasini, R. P. Town, S. V. Weber, P. J. Wegner, K. Widman, M. Wilke, D. C. Wilson, C. B. Yeamans, and A. Zylstra, Phys. Plasmas 20, 070501 (2013).

${ }^{46}$ T. R. Boehly, D. L. Brown, R. S. Craxton, R. L. Keck, J. P. Knauer, J. H. Kelly, T. J. Kessler, S. A. Kumpan, S. J. Loucks, S. A. Letzring, F. J. Marshall, R. L. McCrory, S. F. B. Morse, W. Seka, J. M. Soures, and C. P. Verdon, Opt. Commun. 133, 495 (1997).

${ }^{47}$ K. Anderson and R. Betti, Phys. Plasmas 11, 5 (2004).
${ }^{48}$ Laser Coherence Control: Technology and Applications, edited by H. T. Powell and T. J. Kessler (SPIE, Bellingham, WA, 1993), p. 95.

${ }^{49}$ Y. Lin, T. J. Kessler, and G. N. Lawrence, Opt. Lett. 21, 1703 (1996).

${ }^{50} \mathrm{~K}$. Tsubakimoto, M. Nakatsuka, H. Nakano, T. Kanabe, T. Jitsuno, and S. Nakai, Opt. Commun. 91, 9 (1992).

${ }^{51}$ K. Tsubakimoto, T. Jitsuno, N. Miyanaga, M. Nakatsuda, T. Kanabe, and S. Nakai, Opt. Commun. 103, 185 (1993).

${ }^{52}$ T. E. Gunderman, J.-C. Lee, T. J. Kessler, S. D. Jacobs, D. J. Smith, and S. Skupsky, in Conference on Lasers and Electro-Optics (Optical Society of America, Washington, DC, 1990), Vol. 7, p. 354.

${ }^{53}$ V. Y. Glebov, D. D. Meyerhofer, C. Stoeckl, and J. D. Zuegel, Rev. Sci. Instrum. 72, 824 (2001).

${ }^{54}$ R. A. Lerche, D. W. Phillion, and G. L. Tietbohl, Rev. Sci. Instrum. 66, 933 (1995).

${ }^{55}$ R. Florido, R. C. Mancini, T. Nagayama, R. Tommasini, J. A. Delettrez, S. P. Regan, V. A. Smalyuk, R. Rodríguez, and J. M. Gil, High Energy Density Phys. 6, 70 (2010)

${ }^{56}$ J. Delettrez, R. Epstein, M. C. Richardson, P. A. Jaanimagi, and B. L. Henke, Phys. Rev. A 36, 3926 (1987).

${ }^{57}$ V. N. Goncharov, O. V. Gotchev, E. Vianello, T. R. Boehly, J. P. Knauer, P. W. McKenty, P. B. Radha, S. P. Regan, T. C. Sangster, S. Skupsky, V. A. Smalyuk, R. Betti, R. L. McCrory, D. D. Meyerhofer, and C. CherfilsClérouin, Phys. Plasmas 13, 012702 (2006).

${ }^{58}$ I. V. Igumenshchev, W. Seka, D. H. Edgell, D. T. Michel, D. H. Froula, V. N. Goncharov, R. S. Craxton, L. Divol, R. Epstein, R. Follett, J. H. Kelly, T. Z. Kosc, A. V. Maximov, R. L. McCrory, D. D. Meyerhofer, P. Michel, J. F. Myatt, T. C. Sangster, A. Shvydky, S. Skupsky, and C. Stoeckl, Phys. Plasmas 19, 056314 (2012).

${ }^{59}$ J. J. MacFarlane, I. E. Golovkin, R. C. Mancini, L. A. Welser, J. E. Bailey, J. A. Koch, T. A. Mehlhorn, G. A. Rochau, P. Wang, and P. Woodruff, Phys. Rev. E 72, 066403 (2005).

${ }^{60}$ R. Florido, R. Rodríguez, J. M. Gil, J. G. Rubiano, P. Martel, E. Mínguez, and R. C. Mancini, Phys. Rev. E 80, 056402 (2009); R. Rodríguez, R. Florido, J. M. Gil, J. G. Rubiano, D. Suárez, P. Martel, E. Mínguez, and R. C. Mancini, Commun. Comput. Phys. 8, 185 (2010).

${ }^{61}$ T. J. Burris-Mog, R. C. Mancini, J. E. Bailey, G. A. Chandler, G. Rochau, G. Dunham, P. W. Lake, K. Peterson, S. A. Slutz, T. A. Mehlhorn, I. E. Golovkin, and J. J. MacFarlane, J. Quant. Spectrosc. Radiat. Transfer 99, 120 (2006).

${ }^{62}$ M. F. Gu, Can. J. Phys. 86, 675 (2008).

${ }^{63}$ J. Bauche, C. Bauche-Arnoult, and M. Klapisch, Adv. At. Mol. Phys. 23, 131 (1988)

${ }^{64}$ R. C. Mancini, R. F. Joyce, and C. F. Hooper, Jr., J. Phys. B: At. Mol. Phys. 20, 2975 (1987).

${ }^{65}$ L. A. Woltz and C. F. Hooper, Jr., Phys. Rev. A 38, 4766 (1988).

${ }^{66}$ R. C. Mancini, D. P. Kilcrease, L. A. Woltz, and C. F. Hooper, Jr., Comput. Phys. Commun. 63, 314 (1991).

${ }^{67}$ C. A. Iglesias, H. E. DeWitt, J. L. Lebowitz, D. MacGowan, and W. B. Hubbard, Phys. Rev. A 31, 1698 (1985).

${ }^{68}$ H. A. Kramers, Philos. Mag. 46, 836 (1923).

${ }^{69}$ N. H. Magee, in 14th Topical Conference on Atomic Processes in Plasmas, edited by J. S. Cohen, S. Mazevet, and D. P. Kilcrease (AIP Publishing, New York, 2004), pp. 168-179.

${ }^{70}$ E. J. Iglesias and H. R. Griem, J. Quant. Spectrosc. Radiat. Transfer 55, 383 (1996).

${ }^{71}$ Numerical Recipes: The Art of Scientific Computing, 3rd ed. (Cambridge University Press, 2007).

${ }^{72}$ I. E. Golovkin and R. C. Mancini, J. Quant. Spectrosc. Radiat. Transfer 65, 273 (2000).

${ }^{73} \mathrm{~S}$. Atzeni and J. Meyer-Ter-Vehn, The Physics of Inertial Fusion (Oxford University Press, 2004).

${ }^{74}$ J. Lindl, Phys. Plasmas 2, 3933 (1995). 